Aus der Abteilung Kardiologie und Pneumologie ( Prof. Dr. med. G. Hasenfuß )

im Zentrum Innere Medizin

der Medizinischen Fakultät der Universität Göttingen

\title{
Prognostische Bedeutung der Mikroneurographie in Bezug auf Morbidität und Mortalität bei chronisch obstruktiver Lungenerkrankung und Herzinsuffizienz
}

\author{
INAUGURAL - DISSERTATION \\ zur Erlangung des Doktorgrades \\ der Medizinischen Fakultät der \\ Georg-August-Universität zu Göttingen
}

vorgelegt von

Stephan F. Klarner

aus

Warburg

Göttingen 2012 
Dekan:

1. Berichterstatter:

2. Berichterstatter:

3. Berichterstatter:
Prof. Dr. rer. nat. H. K. Kroemer

Prof. Dr. med. S. Andreas

Prof. Dr. med. J. Hinz

Prof. Dr. med. R. Seipelt

Tag der mündlichen Prüfung: 12.08.2013 


\section{Inhaltsverzeichnis}

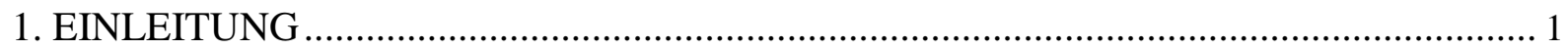

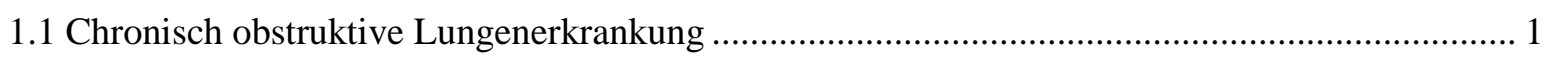

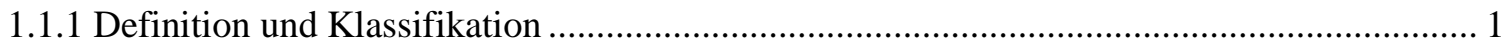

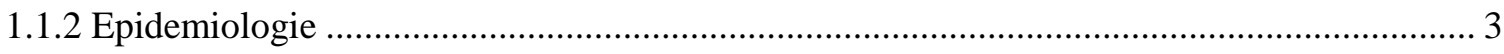

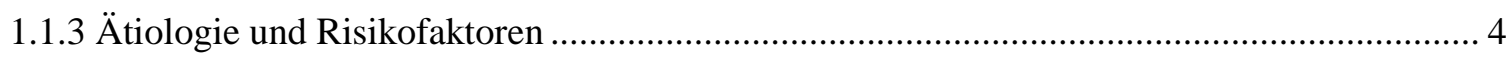

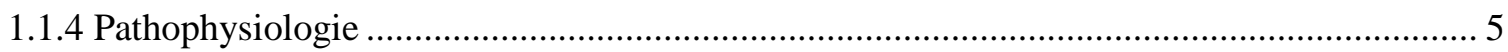

1.2 Bedeutung des vegetativen Nervensystems bei chronisch obstruktiver Lungenerkrankung und

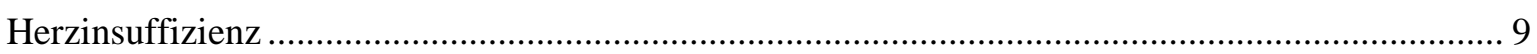

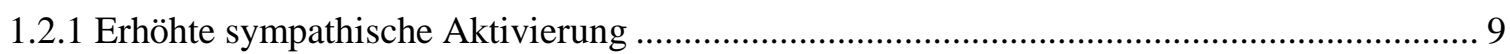

1.2.2 Ätiologie und Pathogenese der sympathischen Aktivierung ............................................ 10

1.2.3 Konsequenzen der erhöhten sympathischen Aktivierung................................................... 16

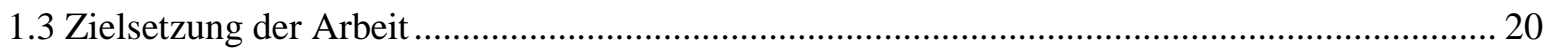

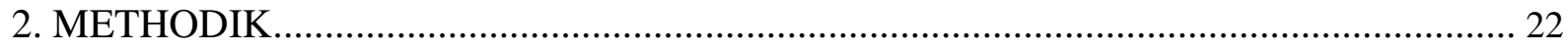

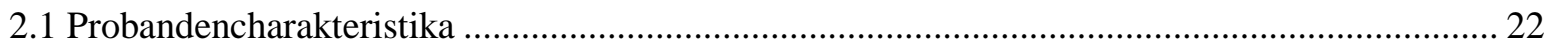

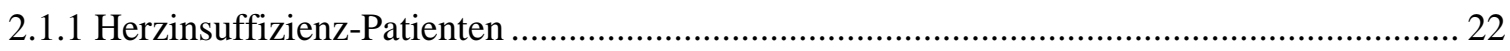

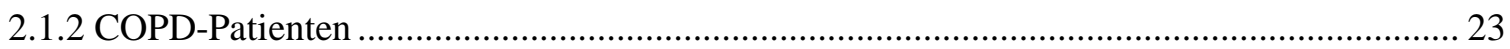

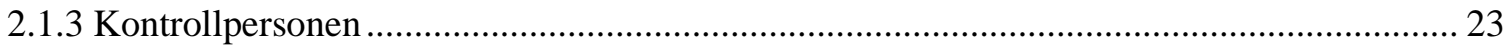

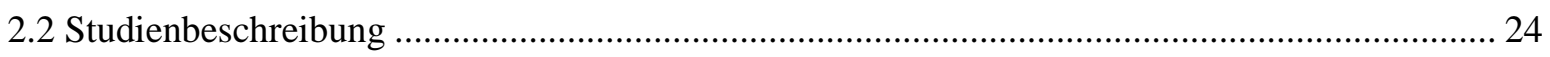

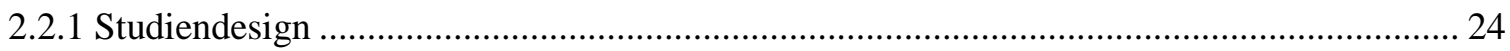

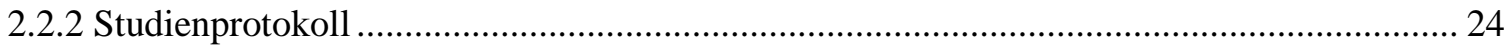

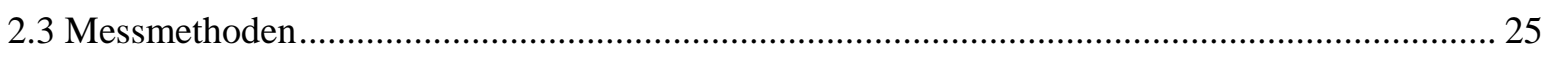

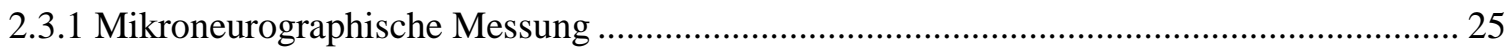

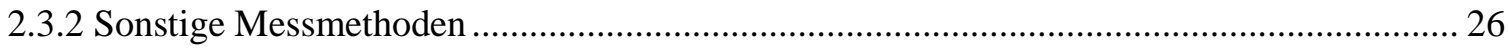

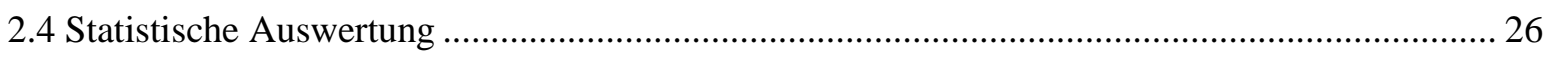

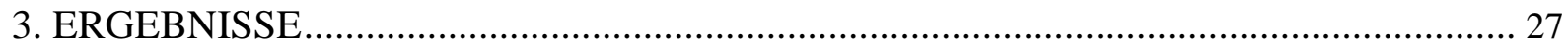

3. 1 Allgemeine statistische Charakteristika der Probandengruppen ................................................ 27

3.1.1 Herzinsuffizienz-Patienten versus Kontrollgruppe ............................................................ 27

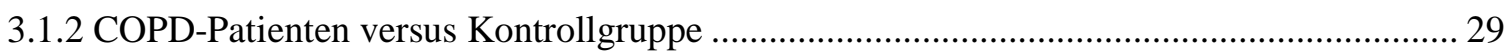




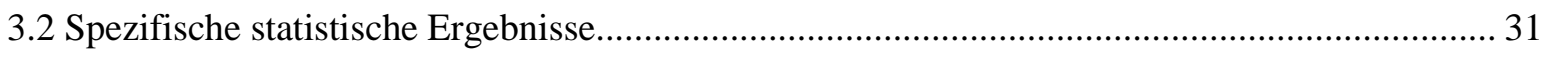

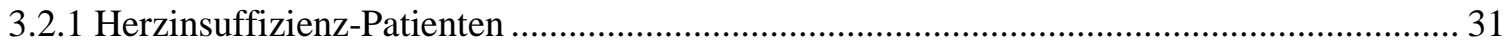

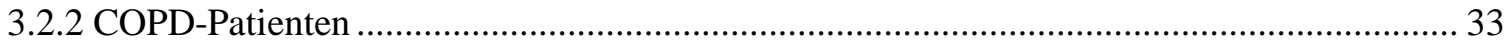

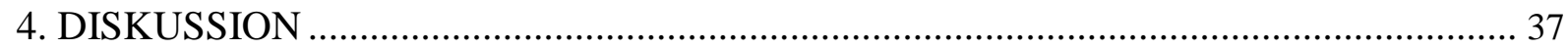

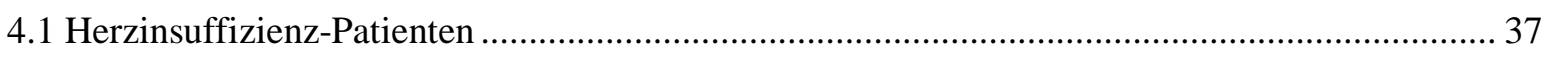

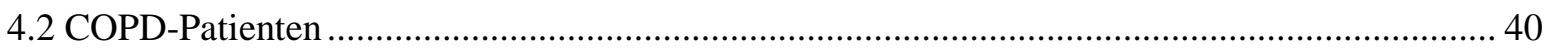

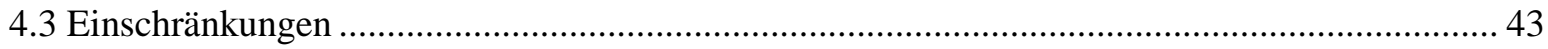

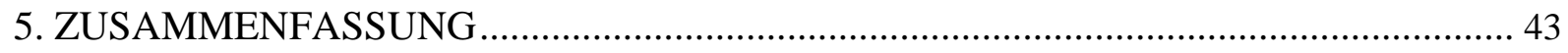

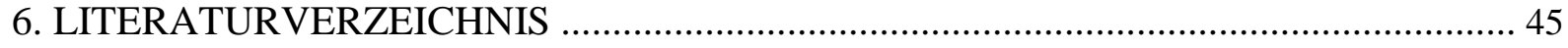




\section{EINLEITUNG}

\subsection{Chronisch obstruktive Lungenerkrankung}

\subsubsection{Definition und Klassifikation}

Die chronisch obstruktive Lungenerkrankung (COPD) ist nach der Global Initiative for Chronic Obstructive Lung Disease (GOLD, Rabe et al. 2007) eine in aller Regel progressive Erkrankung, die auch nach Gabe von Bronchodilatatoren durch eine nicht vollständig reversible Atemwegsobstruktion charakterisiert ist. Die Atemwegsobstruktion wird durch eine Mischung aus obstruktiver Bronchiolitis und parenchymatöser Schädigung verursacht und ist dabei mit einer abnormalen inflammatorischen Antwort der Lunge auf gesundheitsschädliche Partikel oder Gase assoziiert. Darüber hinaus bestehen signifikante extrapulmonale Effekte. Charakteristische Symptome der COPD sind chronische und progressive Dyspnoe, Husten und Sputumproduktion (Rabe et al. 2007).

Die Diagnosestellung und Sicherung einer COPD erfolgt neben der klinischen Diagnose über die Spirometrie. Dabei ist von einer COPD auszugehen, wenn das postbronchodilatatorische forcierte exspiratorische Einsekundenvolumen $\left(\mathrm{FEV}_{1}\right)$ unter $80 \%$ des individuellen Solls beträgt und gleichzeitig $\mathrm{FEV}_{1} / \mathrm{FVC}<70 \%$ vorliegt. Die Stadieneinteilung erfolgt nach GOLD anhand der Spirometrie in vier Stufen (Tabelle 1). 
Tabelle 1: Stadieneinteilung der Chronisch obstruktiven Lungenerkrankung (COPD) nach GOLD (Rabe et al. 2007)

\begin{tabular}{|c|c|}
\hline Stadium & Spirometrie* und häufige Symptome \\
\hline Stadium I - milde COPD & $\begin{array}{l}\mathrm{FEV}_{1} / \mathrm{FVC}<70 \% \\
\mathrm{FEV}_{1}>/=80 \% \\
\text { fakultativ chronischer Husten und Sputumproduktion }\end{array}$ \\
\hline Stadium II - moderate COPD & $\begin{array}{l}\mathrm{FEV}_{1} / \mathrm{FVC}<70 \% \\
50 \%</=\mathrm{FEV}_{1}<80 \% \\
\text { Dyspnoe typischerweise bei Belastung, fakultativ } \\
\text { chronischer Husten }\end{array}$ \\
\hline Stadium III - schwere COPD & $\begin{array}{l}\mathrm{FEV}_{1} / \mathrm{FVC}<70 \% \\
30 \%</=\mathrm{FEV}_{1}<50 \% \\
\text { Kurzatmigkeit, reduzierte körperliche Belastbarkeit, } \\
\text { Erschöpfung, wiederholte Exazerbationen }\end{array}$ \\
\hline Stadium IV - sehr schwere COPD & $\begin{array}{l}\mathrm{FEV}_{1} / \mathrm{FVC}<70 \% \\
\mathrm{FEV}_{1}<30 \% \text { oder } \\
\mathrm{FEV}_{1}<50 \% \text { mit chronisch-respiratorischer } \\
\text { Insuffizienz; } \\
\text { Kurzatmigkeit, reduzierte körperliche Belastbarkeit, } \\
\text { Erschöpfung, wiederholte Exazerbationen, eventuell } \\
\text { Zeichen eines Cor pulmonale }\end{array}$ \\
\hline
\end{tabular}

*FVC und $\mathrm{FEV}_{1}$ bei postbronchodilatatorischer Spirometrie

$\mathrm{FEV}_{1}$ : forciertes exspiratorisches Einsekundenvolumen; FVC: forcierte Vitalkapazität; chronisch-respiratorische Insuffizienz: arterieller Sauerstoffpartialdruck $\left(\mathrm{PaO}_{2}\right)<8.0 \mathrm{kPa} \quad(60 \mathrm{mmHg})$ mit oder ohne Erhöhung des arteriellen Kohlendioxidpartialdruckes $\left(\mathrm{PaCO}_{2}\right)$ 


\subsubsection{Epidemiologie}

Aktuell wird die Prävalenz der COPD im GOLD-Stadium II oder höher je nach Studie und der zugrunde liegenden Daten weltweit auf 8 bis 22\% der über 40-jährigen Bevölkerung geschätzt (Buist et al. 2007; Gershon et al. 2011). Dabei ist zu beachten, dass die Daten zur weltweiten Prävalenz der COPD aufgrund unterschiedlicher Kriterien, Definitionen und der Verfügbarkeit diagnostischer Methoden zum Teil erheblichen Schwankungen unterliegen (Halbert et al. 2003; Halbert et al. 2006).

Die Erkrankung geht mit einer starken Beeinträchtigungen der Lebensqualität durch Faktoren, wie die Luftnot, die Sauerstoff-Langzeit-Behandlung, die Frequenz der Notfallaufnahmen sowie die Häufigkeit der Krankenhausaufenthalte insgesamt einher (Garrido PC et al. 2006). Darüber hinaus ist die COPD mit einer Reihe von Komorbiditäten, wie kardiovaskulären Störungen, Bronchialkarzinomen, Lungeninfektionenen, thrombembolischen Ereignissen, Asthma, Hypertonie, Osteoporose, Arthralgien, gastroduodenalen Ulzera, Depressionen und Angststörungen assoziiert (Raherison und Girodet 2009).

Die COPD ist mit annäherungsweise 2,75 Millionen Toten pro Jahr die fünfthäufigste Todesursache weltweit (Viegi et al. 2007). Es ist davon auszugehen, dass sich dieser Anteil in den nächsten Jahren durch den fortgesetzten weltweiten Tabakkonsum und den demographischen Wandel in vielen Ländern weiter vergrößern wird, sodass die COPD im Jahre 2020 die dritthäufigste Todesursache darstellen könnte (Lopez et al. 2006; Murray und Lopez 1997). Dabei wird die Rolle der Erkrankung in Bezug auf die Mortalität immer noch unterschätzt, da sie häufig nicht als eigentliche Todesursache erkannt wird und über Interaktionen mit assoziierten Erkrankungen zu letalen Verläufen beiträgt (Hansell et al. 2003; Jensen et al. 2006; Sin et al. 2006).

Die Behandlung und der Ausfall der krankheitsbedingten Erwerbsunfähigkeit in der Europäischen Union verursachte im Jahr 2001 geschätzte Kosten von 38,7 Milliarden Euro und belasten somit die Gesundheitssysteme erheblich (Viegi et al. 2007). Auch hier ist davon auszugehen, dass indirekte Kosten aufgrund beeinträchtigter Erwerbsfähigkeit pflegender Angehöriger den ökonomischen Schaden weiter vergrößern (Viegi et al. 2007).

Die Behandlung der chronisch obstruktiven Lungenerkrankung stellt damit eine sowohl medizinische als auch ökonomische wachsende Herausforderung der Zukunft dar, die jedoch in den gesundheitspolitischen Betrachtungen und Bemühungen bisher nicht mit entsprechendem Stellenwert beachtet wird. 


\subsection{3 Ätiologie und Risikofaktoren}

Der Tabakkonsum ist als Hauptrisikofaktor für annähernd 70-90\% der COPD-Fälle verantwortlich. Dabei steigt die Prävalenz in einer Population mit dem Anteil an Rauchern und das persönliche Risiko mit dem kumulativen Zigarettenkonsum der einzelnen Person (de Marco et al. 2004; Halbert et al. 2006). Insgesamt entwickeln dadurch je nach Lebenserwartung und Intensität des Tabakkonsums bis zu 30\% der Raucher im Verlauf ihres Lebens eine COPD (Lokke et al. 2006; Rennard und Vestbo 2006). Neben dem aktiven Tabakrauchen erhöht auch das Passivrauchen die Wahrscheinlichkeit, an einer COPD zu erkranken, um etwa 40\% (Society 2006; Yin et al. 2007).

Durch diesen enormen Einfluss des Tabakrauchs auf die Entstehung und die Progredienz der Erkrankung kommt der Raucherentwöhnung eine Schlüsselposition in der Prävention und Therapie der COPD zu. Eine Tabakabstinenz führt zu einer Verbesserung von Lungenfunktion und Symptomatik und stellt damit die effektivste medizinische Intervention in der Therapie der COPD dar (Andreas et al. 2009; Park und Sin 2011).

Aus der Tatsache, dass nicht alle Raucher eine COPD entwickeln und auch Nichtraucher erkranken lässt sich schließen, dass eine genetische Prädisposition Einfluss auf die Entstehung hat (Molfino 2004). Neben verschiedenen genetischen Polymorphismen, die die Entwicklung einer COPD begünstigen, gilt eine schwere $\alpha_{1}$-Antitrypsin-Defizienz als gesicherter genetischer Faktor für die Entstehung einer COPD mit der Ausprägung eines panlobulären Emphysems (Stoller und Aboussouan 2005).

Als weiterer Risikofaktor für die Entstehung einer COPD gilt eine signifikante berufliche Exposition gegenüber verschiedenen organischen und anorganischen Stäuben und Partikeln in landwirtschaftlichen und industriellen Berufen (Hnizdo et al. 2002). Dabei ist von einem synergistischen Effekt von beruflicher Exposition und Tabakkonsum auszugehen (Blanc et al. 2009).

Des Weiteren gibt es Hinweise darauf, dass eine Luftverschmutzung durch Autoabgase, Stickstoffdioxid, Ozon und Partikelmaterie die Inzidenz der COPD in entsprechend belasteten Gebieten erhöht. Allerdings bedarf es weiterer umfassender epidemiologischer Studien, um eine valide Aussage diesbezüglich zu treffen (Kelly und Fussell 2011).

Die Prävalenz der COPD korreliert invers mit den sozioökonomischen Konditionen. Personen, die einem niedrigeren sozialen Milieu angehören, erkranken mit einer höheren Wahrscheinlichkeit an COPD. Inwieweit dieser Tatsache lediglich eine erhöhte Belastung mit bekannten Risikofaktoren zugrunde liegt oder ob andere schichtspezifische Faktoren hinzukommen, ist noch nicht abschließend geklärt (Rabe et al. 2007). 


\subsubsection{Pathophysiologie}

\subsubsection{Pulmonale Pathophysiologie}

Bei der COPD kommt es, zumeist durch exogene Noxen getriggert, zu einer andauernden Entzündungsreaktion im Lungenparenchym mit damit einhergehenden strukturellen Veränderungen, die letztlich die klinische Symptomatik des Patienten bedingen.

Die chronische Exposition des respiratorischen Epithels und der Alveolen gegenüber Zigarettenrauch führt zu einer erhöhten Permeabilität (Jones et al. 1980), wodurch eine fortwährende Entzündungsreaktion entsteht. Dieser Entzündungsprozess beeinträchtigt durch Schädigung des Flimmerepithels die mukoziliäre Clearance und begünstigt so respiratorische Infekte (Sethi und Murphy 2001).

Darüber hinaus führen die inhalierten Noxen über oxidativen Stress zu einem Ungleichgewicht zwischen Proteasen und Antiproteasen mit gesteigerter proteolytischer Aktivität im Lungengewebe. Dies geschieht einerseits, weil der Entzündungsprozess zur Freisetzung von Oxidanzien und Proteasen aus Makrophagen und Neutrophilen Granulozyten führt, andererseits bewirkt der Zigarettenrauch eine Inaktivierung von Antiproteasen und damit eine verminderte Aktivität des antioxidativen Systems, unter anderem bestehend aus der Glutathion-Peroxidase und der Haemoxygenase-1. Die verminderte Aktivität beider Enzyme korreliert dabei invers mit dem Grad der Obstruktion und Überblähung. Durch den Entzündungsprozess wird daher das initial durch den Zigarettenrauch ausgelöste Ungleichgewicht weiter verstärkt (Rahman et al. 2006).

Die im Rahmen der Entzündungsreaktion freigesetzten Proteasen greifen die extrazelluläre Matrix der Lunge an und führen zur Degeneration der elastischen und kollagenen Fasern (Bourdin et al. 2009; Maestrelli et al. 2003). Durch den Protease-vermittelten Elastinabbau und die Apoptose der Typ-IAlveolarzellen und Endothelzellen entsteht im Endstadium der COPD das Bild eines Emphysems, welches charakterisiert ist durch eine abnormale, permanente Erweiterung der Luftwege distal der Bronchioli terminales und die Destruktion der Alveolarwände (Barnes 2008a; Fischer et al. 2011; National Heart... 1985).

Im Zuge der Entzündungsreaktion findet eine Zytokinausschüttung, unter anderem von Interleukin-8, statt, was zu einem Anstieg der Neutrophilen Granulozyten führt, deren Elastase eine zusätzliche Aktivierung von Makrophagen und Becherzellen bewirkt. Die erhöhte Sekretproduktion der Becherzellen ist neben den strukturellen Veränderungen der Luftwege für den obstruktiven Charakter der Erkrankung verantwortlich (Barnes 2008b). Daher korreliert auch das Ausmaß der Neutrophilenkonzentration im Sputum mit der Schwere der Erkrankung (Keatings et al. 1996). Neben 
den Makrophagen und Neutrophilen Granulozyten spielen auch B-Lymphozyten und insbesondere CD8 ${ }^{+}$-T-Lymphozyten eine wichtige Rolle im Entzündungsgeschehen der COPD.

Durch die Ausschüttung von transforming growth factor- $\beta$ (TGF- $\beta$ ) aus Makrophagen kommt es zur Fibrose der kleinen Luftwege mit einhergehender Wandverdickung und Einengung des Lumens (Barnes 2008a).

Die oben beschriebenen pathophysiologischen Veränderungen führen in ihrer Gesamtheit zu erheblichen Einschränkungen der Lungenfunktion. Bei COPD-Patienten findet aufgrund der Obstruktion der kleinen Atemwege ein verstärkter Abfall der FEV ${ }_{1}$ statt, der sich wesentlich schneller als physiologisch vollzieht (Hogg 2004). Zusätzlich ist die Diffusionskapazität aufgrund der Rarefizierung der Alveolen und der verdickten Bronchioli vermindert und das Residualvolumen erhöht. Es kommt zunehmend zum Abfall des maximalen exspiratorischen Flusses, unter anderem bedingt durch die erniedrigten elastischen Rückstellkräfte der Lunge als Folge des Abbaus elastischer Fasern (Mead et al. 1967).

\subsubsection{Extrapulmonale Pathophysiologie}

Im Zuge der Erkrankung kommt es zu signifikanten extrapulmonalen Effekten, unter anderem zu einer systemischen Entzündungsreaktion, die sich durch eine Erhöhung von proinflammatorischen Zytokinen, von Akute-Phase-Proteinen und durch funktionelle Veränderungen sowie einen Anstieg zirkulierender Immunzellen bemerkbar macht (Barnes und Celli 2009). Dabei sind hohe Spiegel der

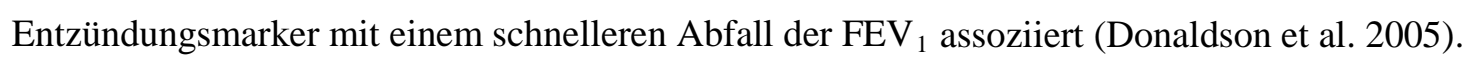

Als wichtiges proinflammatorisches Zytokin ist Interleukin-6 zu nennen, dessen Erhöhung möglicherweise im Zusammenhang mit Skelettmuskelschwäche und Herzschwäche steht (Janssen et al. 2005). Ferner kommt es zur Konzentrationssteigerung des Tumor-Nekrose-Faktor- $\alpha$ (TNF- $\alpha$ ), insbesondere bei rauchenden COPD-Patienten, und Interleukin-8 (IL-8) (Tanni et al. 2010). Beide Zytokine tragen zu verstärkter Kachexie, Skelettmuskelatrophie und Muskelschwäche bei (Spruit et al. 2003; Tracey et al. 1988). Interleukin-8 aktiviert, wie oben bereits beschrieben, die neutrophile Immunreaktion und unterstützt damit die Entzündungsreaktion in der Lunge (Beeh et al. 2003).

Als weiterer Marker des systemischen Entzündungsgeschehens lässt sich eine Erhöhung der AkutePhase-Proteine C-reaktives Protein (CRP), Fibrinogen, Serum Amyloid A und Surfactant Protein D ausmachen (Barnes und Celli 2009). Der Anstieg der proinflammatorischen Zytokine und der AkutePhase-Proteine ist dabei während einer akuten Exazerbation der COPD besonders stark (Agusti et al. 2003). Als Folge der systemischen Entzündungsreaktion kommt es zur intensivierten Freisetzung von Leukozyten und Thrombozyten aus dem blutbildenden Knochenmark (Barnes und Celli 2009). 
Die skeletto-muskuläre Dysfunktion und Muskelatrophie, die mit der COPD einhergeht, ist durch ein gestörtes Verhältnis im Proteinmetabolismus zwischen Proteinsynthese und Proteindegeneration charakterisiert. Dabei spielt zum einen die systemische Entzündung mit ihren oben beschriebenen Mediatoren eine Rolle, zum anderen trägt auch ein vermehrter oxidativer und nitrosativer Stress durch eine Erhöhung reaktiver Radikale und/oder verminderte antioxidative Kapazitäten bei (Agusti et al. 2004; Debigare et al. 2001; Supinski und Callahan 2007). Die Folgen dieses Prozesses, insbesondere einer Manifestation der Muskelschwäche am Diaphragma führen zu einer weiteren Verschlechterung der respiratorischen Kompetenz (Ottenheijm et al. 2007; Ottenheijm et al. 2008; Testelmans et al. 2010). Das Ausmaß des Muskelabbaus hat dabei signifikanten Einfluss sowohl auf Morbidität als auch auf Mortalität der COPD-Patienten (Barnes und Celli 2009).

\subsubsection{Assoziierte Komorbiditäten}

Bei Patienten, die unter der COPD leiden, lassen sich im erhöhten Maße Erkrankungen feststellen, die in ihrer Pathogenese durch die Pathophysiologie der COPD begünstigt werden oder durch gemeinsame Risikofaktoren vermehrt auftreten und die Morbidität des Patienten zusätzlich negativ beeinflussen. Die wichtigsten assoziierten Erkrankungen sind die pulmonal arterielle Hypertonie, die Anämie, die Osteoporose und die koronare Herzerkrankung.

Im Laufe der COPD entsteht eine endotheliale Dysfunktion in den Lungenarterien, bei der es zu einer verminderten Ausschüttung von vasoaktiven Mediatoren wie Stickstoffmonoxid und Prostacyclin kommt. Dies führt, auch verstärkt durch die Hypoxie, zu pulmonalarterieller Vasokonstriktion mit Erhöhung des Gefäßdrucks. Im weiteren Verlauf bewirkt die Ausschüttung von Zytokinen und Wachstumsfaktoren, wie dem Vascular endothelial growth factor (VEGF), in Kombination mit der herrschenden Hypoxie einen irreversiblen Umbau des pulmonalen Gefäßsystems (Barbera et al. 2003; Tuder et al. 1995). Pathohistologisch lässt sich in leichteren und moderaten Stadien der COPD zunächst eine Fibrose und Proliferation glatter Muskelzellen erkennen, die durch eine Verdickung der Gefäßwandintima der pulmonalen Arteriolen zur konsekutiven Einengung des Lumens führt. Bei schwereren Stadien der COPD finden darüber hinaus auch Veränderungen zirkulärer glatter Muskelzellen der Gefäßwandmedia statt (Wright JL et al. 1992). Als Folge kann sich eine pulmonal arterielle Hypertonie mit Rechtsherzbelastung entwickeln, die im Endstadium mit dem Bild des Cor pulmonale imponiert.

Auffällig ist eine hohe Prävalenz einer Anämie bei, je nach Studie, bis zu 15-30\% der COPDPatienten (Barnes und Celli 2009). John et al. diskutieren als Ursache der zumeist normochromen, normozytären Anämie die chronisch systemische Entzündungsreaktion, die auf die regulatorisch 
erhöhte endogene Erythropoetin-Ausschüttung nicht anspricht (John et al. 2005). Anämische COPDPatienten neigen zu erhöhter Dyspnoe, erniedrigter funktionaler Kapazität und erhöhter Mortalität (Boutou et al. 2011; Cote et al. 2007). Es gibt Hinweise, dass eine therapeutische Korrektur der Anämie die Atemarbeit verringert und die Beatmungsentwöhnung erleichtert (Schonhofer et al. 1998a; Schonhofer et al. 1998b).

Die Prävalenz der Osteoporose bei COPD-Patienten variiert je nach Studie, Patientenkollektiv und diagnostischen Kriterien zwischen 9\% und 69\% bei einem Mittelwert von 35,1\% und ist gegenüber der gleichalten Normalbevölkerung insgesamt signifikant erhöht (Graat-Verboom et al. 2009). Das vermehrte Auftreten der Osteoporose lässt sich neben einhergehenden, verbreiteten Lebensstilcharakteristika, wie Inaktivität, schlechte Ernährung und Rauchen, vor allem durch die systemische Entzündungsreaktion und Therapie der COPD erklären. Dabei führen, neben einer Vielzahl anderer Mechanismen, proinflammatorische Zytokine, wie beispielsweise der TNF- $\alpha$, zur Stimulation der Osteoklastendifferenzierung und -aktivierung und damit zu einem vermehrten Knochenabbau (Hardy und Cooper 2009). Eine systemisch relevante Therapie mit Glukokortikoiden kann bei entsprechender Dauer über eine Beeinflussung der Osteoblastenreplikation und -aktivität, der Induktion von Apoptose bei Osteoblasten und Osteozyten und der Förderung der Osteoklastenproliferation zu einer Glukokortikoid-induzierten Osteoporose führen (Canalis et al. 2007). Da das Vorliegen einer Osteoporose die Wahrscheinlichkeit für Frakturen, insbesondere für Wirbelkompressionsfrakturen, erhöht und diese, selbst wenn sie klinisch asymptomatisch sind, signifikanten Einfluss auf die Leistung und den Abfall der Lungenfunktion haben und somit auch auf die Häufigkeit von Exazerbationen, ist es wichtig frühzeitig eine Osteoporosediagnostik und -therapie durchzuführen, um so der Verkomplizierung der systemischen Erkrankung der COPD entgegenzuwirken (Lehouck et al. 2011).

Epidemiologisch konnte gezeigt werden, dass Patienten mit einer erniedrigten $\mathrm{FEV}_{1}$ unabhängig von Alter, Rauchgewohnheiten und Geschlecht ein signifikant erhöhtes Risiko haben, einen letalen Myokardinfarkt zu erleiden (Sin et al. 2005). Zudem haben Patienten mit dem GOLD COPD-Grad 3 und 4 eine erhöhte Prävalenz von Hypertonie und kardiovaskulären Erkrankungen. Dies könnte zum Teil an gemeinsamen Risikofaktoren liegen, aber auch begünstigt werden durch die erhöhte sympathische Aktivierung und die systemische Entzündungsreaktion, welche beide eine Rolle in der Pathogenese der koronaren Herzerkrankung mit Arteriosklerose spielen (Andreas et al. 2005; Barnes und Celli 2009; Mannino et al. 2008; Rodrigues et al. 2010; Tsuji et al. 1996). 


\subsection{Bedeutung des vegetativen Nervensystems bei chronisch obstruktiver Lungenerkrankung und Herzinsuffizienz}

\subsubsection{Erhöhte sympathische Aktivierung}

Sowohl bei der chronisch obstruktiven Lungenerkrankung als auch bei der chronischen Herzinsuffizienz konnte in verschiedenen Studien gezeigt werden, dass bei Patienten, im Vergleich mit gesunden Kontrollpersonen, eine erniedrigte Herzfrequenzvariabilität als Marker einer kardialen sympathischen Aktivierung vorliegt. Bei COPD-Patienten war diese zum Teil durch Sauerstoffsupplementation reversibel (Bartels et al. 2000; Scalvini et al. 1999; Volterrani et al. 1994). Der Abfall der Herzfrequenzvariabilität korreliert bei der COPD, wie auch der chronischen Herzinsuffizienz, mit der Schwere der Erkrankung und scheint nicht durch anticholinerge oder adrenerge Medikation beeinflusst zu werden (Bedard et al. 2010; Ponikowski et al. 1997a).

Es ist schon länger hinreichend bekannt, dass bei Patienten mit chronischer Herzinsuffizienz auch über die Mikroneurographie eine signifikant erhöhte periphere sympathische Aktivierung gemessen werden kann und diese mit der Schwere der Erkrankung zunimmt (Ferguson et al. 1990; Negrao et al. 2001). Im Jahre 2001 konnten Heindl et al. mittels Mikroneurographie bei hypoxischen COPD-Patienten zeigen, dass ebenfalls eine signifikant erhöhte periphere sympathische Aktivierung vorliegt, die im Vergleich mit gesunden Kontrollpersonen ungefähr doppelt so hoch war und sich nicht über eine begleitende Medikation erklären ließ. Dabei konnte bei den Patienten durch die nasale Kurzzeitapplikation von 4 l O2/min, im Gegensatz zu den Kontrollpersonen, ein moderater Abfall der peripheren sympathischen Aktivierung beobachtet werden, allerdings kam es nicht zur Normalisierung selbiger (Heindl et al. 2001b).

Im Jahr 2008 konnten Raupach et al. auch bei normoxischen COPD-Patienten, im Vergleich zu gesunden Kontrollpersonen, mikroneurographisch eine ebenfalls fast doppelt so hohe sympathische Aktivierung verifizieren (Raupach et al. 2008).

Diese beiden Studien, in denen erstmals die erhöhte sympathische Aktivität bei COPD mikroneurographisch nachgewiesen wurde, stammen aus derselben Arbeitsgruppe der Abteilung für Kardiologie und Pneumonologie der Universitätsmedizin Göttingen und dienten unter anderem als Grundlage für diese Studie.

In einigen Studien konnte nachgewiesen werden, dass normoxische Patienten, die sich im Endstadium eines Emphysems befanden, fast doppelt so hohe Plasma-Noradrenalin-Werte hatten wie gesunde 
Kontrollpersonen (Hofford et al. 1990). Bei COPD-Patienten konnte zusätzlich ein erhöhter myokardialer Noradrenalinumsatz als Zeichen einer systemischen Überaktivität des Sympathikus gemessen werden (Sakamaki et al. 1999).

In einer anderen Studie konnte bei COPD-Patienten eine erhöhte Noradrenalinexkretion im Urin festgestellt werden, die positiv mit einer nächtlichen Sauerstoffsättigung von unter 85\% korrelierte (Bratel et al. 2000).

Zusammenfassend kann durch die derzeitige Studienlage sowohl bei der chronischen Herzinsuffizienz, als auch der COPD ein pathologisch erhöhter Sympathikotonus festgestellt werden.

\subsection{2 Ätiologie und Pathogenese der sympathischen Aktivierung}

\subsubsection{Herzinsuffizienz}

Bei der chronischen Herzinsuffizienz steht die erhöhte sympathische Aktivierung im engen Verhältnis zu Modifikationen in kardiovaskulären Reflexen. Dabei sind die Sympathikus-hemmenden arteriellen Barorezeptorreflexe signifikant erniedrigt und die Sympathikus-fördernden Reflexe, wie kardiale afferente Reflexe und arterielle Chemorezeptorreflexe, verstärkt (Watson et al. 2006).

\subsection{Arterieller Barorezeptorreflex}

Die arteriellen Barorezeptoren sind im Aortenbogen und Karotissinus lokalisiert und bewirken bei Stimulation über einen Reflexbogen eine Hemmung des Sympathikotonus. Bei der Herzinsuffizienz ist dieser Reflex nachweislich signifikant erniedrigt und kann infolgedessen zur erhöhten sympathischen Aktivierung beitragen (Jung et al. 1995; Wang W et al. 1990; Wang W et al. 1991; Wang W et al. 2004; White 1981).

\subsection{Kardialer afferenter Reflex}

Ein weiterer sympatho-exzitatorischer Reflex läuft über kardiale afferente Fasern, die mit sensorischen Enden in den Ventrikeln und der thorakalen Aorta liegen. Diese können sowohl über Bradykinin, Adenosin und Sauerstoffperoxid als chemische Mediatoren einer myokardialen Ischämie, als auch mechanisch stimuliert werden (Malliani und Montano 2002). Dieser kardiale sympathisch-afferente Reflex erhöht daher bei der Herzinsuffizienz den sympathischen Ausstoss (Du und Chen 2007).

\subsection{Chemorezeptorreflex}

Die Chemorezeptoren liegen peripher in den Glomerula aortica und carotica, sowie zentral im Hirnstamm. Die peripheren Rezeptoren reagieren dabei vor allem auf eine hypoxische Situation, während die zentralen Rezeptoren besonders auf hyperkapnische Zustände mit einer Aktivierung des Sympathikus reagieren (Marshall 1994). Eine erhöhte Sensitivität der peripheren und zentralen 
Chemorezeptorreflexe konnte sowohl im Tierexperiment als auch bei Herzinsuffizienz-Patienten nachgewiesen werden (Chua et al. 1997; Narkiewicz et al. 1999). Darüber hinaus haben Herzinsuffizienz-Patienten, die zusätzlich unter einer Anämie leiden, eine signifikant erhöhte sympathische Aktivierung gegenüber Patienten, die lediglich herzinsuffizient sind (Franchitto et al. 2010). Dies bestätigt ebenfalls den Einfluss der Chemorezeptoren auf den sympathischen Tonus.

Die Afferenzen dieser drei Reflexe interagieren unter anderem im Nucleus tractus solitarii miteinander (Machado et al. 1997; Tjen et al. 1997). Dabei scheint ein Antagonismus zwischen den peripheren Chemorezeptorreflex und dem arteriellen Baroreflex bei Gesunden, wie auch bei Herzinsuffizienten vorzuliegen. Eine Hemmung des Chemoreflexes führt daher zu einer Verbesserung des pathologisch veränderten arteriellen Baroreflexes (Ponikowski et al. 1997b; Somers et al. 1991).

\subsection{Zentrale Modulation der sympathischen Aktivierung}

Als wichtiger Faktor trägt offenbar die zentrale Modulation der afferenten neuronalen und humoralen Signale zur sympathischen Aktivierung, dem kardialen Remodeling und der Dysfunktion bei. Eine erhebliche Funktion bei der zentralen Verarbeitung und den pathophysiologischen Konsequenzen nehmen dabei Angiotensinrezeptoren ein. Diese sind in hoher Dichte in den zirkumventrikulären Organen verteilt, insbesondere in den Hirnstammkernen, wie dem Nucleus paraventricularis, welcher als wichtiger integrativer Bestandteil der zentralen Modulation sympathischer Afferenzen zu sehen ist (Kenney et al. 2003; Wright JW und Harding 1992).

Welche spezifischen Faktoren genau miteinander interagieren und zur Aktivierung der zentralen Signaltransduktionswege mit sympathischer Aktivierung führen, ist bisher noch nicht abschließend erforscht worden. Allerdings konnte in verschiedenen Studien im Tierexperiment aufgezeigt werden, dass unter anderen drei Mechanismen auf die zentrale Verarbeitung einwirken.

Zum einen werden vegetative kardiale afferente Fasern aktiviert und fördern über $\mathrm{AT}_{1}$-Rezeptoren im Nucleus paraventricularis des Hypothalamus die sympathische Aktivierung (Zhu et al. 2004).

Als weiterer Mechanismus wird die erhöhte Aktivität des peripheren Renin-Angiotensin-AldosteronSystems (RAAS) mit den Hormonen Angiotensin II und Aldosteron gesehen (Lal et al. 2004; Zhang et al. 2002).

Angiotensin II bewirkt über verschiedene Signalwege eine Erhöhung des zentralen sympathischen Ausflusses (Garrido AM und Griendling 2009; Li YF et al. 2006; Reid 1992).

Bei Ratten mit Zustand nach Myokardinfarkt konnte eine zentrale intraventrikuläre Blockade der mineralkortikoiden Rezeptoren durch die Injektion von Spironolacton gegenüber der Kontrollgruppe die sympathische Aktivierung verhindern, die arterielle Baroreflex-Funktion erhalten und die kardiale 
Funktion verbessern (Huang und Leenen 2005). Ein ähnlicher Effekt konnte durch die zentrale Blockade von $\mathrm{AT}_{1}$-Rezeptoren durch Losartan beobachtet werden (Leenen 2007; Leenen et al. 1995). Des Weiteren hatten transgene Ratten, mit einer spezifischen Defizienz von Angiotensinogenen und Angiotensinen im Gehirn, nach einem induzierten Myokardinfarkt eine deutliche Abschwächung des pathologischen Remodelings und damit eine signifikant bessere linksventrikuläre Funktion als die Kontrollgruppe (Lal et al. 2005; Wang W et al. 2004).

Als dritter Mechanismus spielen postinflammatorische Zytokine bei der Erhöhung der zentralen sympathischen Aktivität eine Rolle. Im Zuge eines kardialen Schadens und in der Pathogenese der chronischen Herzinsuffizienz kommt es zur Freisetzung von peripher zirkulierenden Zytokinen, wie TNF- $\alpha$ und IL- $1_{\beta}$. Diese induzieren an perivaskulären und endothelialen Zellen der Bluthirnschranke die Cyclooxygenase-2 ( $\left.\mathrm{COX}_{2}\right)$ und fördern die Synthese von Prostaglandin $\mathrm{E}_{2}$ (Rivest et al. 2000). Prostaglandin $\mathrm{E}_{2}$ ist in der Lage, die Bluthirnschranke zu überwinden und aktiviert Neurone im hypothalamischen Nucleus paraventricularis (Engblom et al. 2002; Ferri und Ferguson 2005). Dies führt zur Ausschüttung von Corticotropin-releasing hormone (CRH) und zur Aktivierung der Hypothalamus-Hypophysen-Nebennierenrinden-Achse, was in einer Erhöhung peripherer Glukokortikoide, Mineralkortikoide, Katecholamine und einer verstärkten sympathischen Nervenaktivität mündet (Fisher 1993; Turnbull und Rivier 1999). Die erhöhten proinflammatorischen Zytokine tragen daher zur verstärkten sympathischen Aktivität bei und interagieren darüber hinaus im Nucleus paraventricularis synergistisch mit Angiotensin II (Shi et al. 2011).

Kang et al. konnten im Tierexperiment zeigen, dass Ratten mit Herzinsuffizienz nach induziertem Myokardinfarkt bei der Behandlung sowohl mit dem Aldosteronantagonisten Eplerenon (oral), als auch mit dem TNF- $\alpha$-Antikörper Etanercept und Pentoxifyllin, eine erniedrigte Plasmakonzentration von TNF- $\alpha$, IL- $1_{\beta}$ und IL-6 haben und die Aktivität der Neurone des Ncl. paraventricularis und der $\mathrm{COX}_{2}$ in den Zellen der Bluthirnschranke vermindert waren, woraus eine erniedrigte sympathische Aktivität resultiert (Guggilam et al. 2008; Kang et al. 2006). Eine verminderte Freisetzung von TNF- $\alpha$ konnten auch Francis et al. durch die zentrale Injektion von Spironolacton zeigen (Francis J et al. 2003).

Diese Beobachtungen machen deutlich, wie stark offenbar das Renin-Angiotensin-Aldosteron-System, die inflammatorischen Komponenten und die erhöhte sympathische Aktivierung in der Pathophysiologie der chronischen Herzinsuffizienz miteinander verzahnt sind. 


\subsection{Individuelle Faktoren der sympathischen Aktivierung}

Neben der funktionellen Schwere der Herzinsuffizienz spielen weitere Faktoren für die Ausprägung der individuellen sympathischen Aktivierung eine Rolle (Floras 2009; Munhoz et al. 2009). In der Bewertung der erhöhten sympathischen Aktivierung muss die Ätiologie der Herzinsuffizienz, Komorbiditäten und konditionelle Eigenschaften der Patienten beachtet werden.

Die Ausprägung der sympathischen Aktivierung ist assoziiert mit der Ätiologie der Herzinsuffizienz. Patienten mit ischämischer Herzinsuffizienz zeigen gegenüber Patienten mit dilatativer Kardiomyopathie eine signifikant höhere sympathische Aktivierung (Notarius et al. 2007).

Der Grad der sympathischen Aktivierung erhöht sich zudem signifikant durch diverse Komorbiditäten, wie beispielsweise das zusätzliche Auftreten eines Schlafapnoe-Syndroms (Spaak et al. 2005).

Ein weiterer Faktor, der sich auf das Ausmaß der individuellen sympathischen Aktivierung auswirkt, stellt die physische Konditionierung des Patienten dar. Ein physisches Training erniedrigt signifikant die sympathische Aktivierung und verbessert die autonomen Reflexe und die abnorme Regulation des RAAS (Li YL et al. 2008; Liu et al. 2000; Roveda et al. 2003). Interessanterweise findet dies zum Teil über eine Normalisierung der pathologisch veränderten zentralen Modulation, unter anderem im Ncl. paraventricularis statt (Brum et al. 2011; Kleiber et al. 2008; Liu et al. 2000; Patel und Zheng 2012; Roveda et al. 2003).

\subsubsection{Chronisch obstruktive Lungenerkrankung}

\subsection{Neuroanatomische Zusammenhänge}

Die neuronalen Kernzentren zur Regulation der Atmung stehen im Hirnstamm in enger anatomischer und funktioneller Verbindung mit der autonomen Kontrolle. Insbesondere die Perzeption respiratorischer Insuffizienz ist in den sensomotorischen Arealen des limbischen Systems repräsentiert, welche regulatorisch auf die autonome Kontrolle einwirken. Darüber hinaus ist der zentrale respiratorische Atemantrieb mit der sympathischen Aktivität im Hirnstamm verbunden (Shields 1993; Zhong et al. 1997).

\subsection{Chemorezeptorreflex}

Eine akute und chronische Hypoxie führt bei gesunden Personen zu einer über die Hypoxie hinaus andauernden neuronalen und humoralen sympathischen Aktivierung mit erhöhten mikroneurographischen Messwerten, Noradrenalin-Konzentrationen und einer Steigerung des Blutdrucks (Calbet 2003; Hansen und Sander 2003; Somers et al. 1989).

Bei COPD-Patienten liegt jedoch auch unter normoxischen Bedingungen eine nachgewiesene sympathische Aktivierung vor, die in dem Ausmaß nicht durch eine Medikation, eine Erkrankung mit bekannter sympathischer Aktivierung oder eine Hyperkapnie zu erklären ist (Heindl et al. 2001b; 
Raupach et al. 2008). Allerdings konnte bei hypoxischen COPD-Patienten durch Erhöhung der inspiratorischen Sauerstoffkonzentration eine mäßige Abnahme der peripheren sympathischen Aktivierung beobachtet werden (Heindl et al. 2001b). Dies spricht für eine additive, synergistische Rolle der Hypoxie bei COPD-Patienten in der Erhöhung der sympathischen Aktivierung und bestätigt die Beobachtungen bei oben beschriebenen anämischen Herzinsuffizienz-Patienten.

\subsection{Muskelmetaboreflex}

Ein Mechanismus, der bei Gesunden nachgewiesen werden konnte und die sympathische Aktivierung fördert, ist der Muskel-Metaboreflex. Aus starker Muskelaktivität resultiert eine Freisetzung von Sauerstoffradikalen und Produkten des anaeroben Stoffwechsels, wie zum Beispiel Adenosin, Prostaglandinen und Lactat, mit einer lokalen Veränderung des metabolischen Milieus und nachgewiesenen Auswirkungen auf die sympathische Aktivierung (Costa und Biaggioni 1994; Notarius et al. 2001; Scott et al. 2003; Scott et al. 2002).

Die Skelettmuskeln und speziell das Diaphragma verfügen über afferente, unmyelinisierte sensorische Typ-III- und -IV-Nervenfasern, deren chemosensitive Nervenendigungen im Interstitium liegen und die einen Reflexbogen darstellen, der in Ruhe und besonders bei Belastung die Wirkung auf das sympathische Nervensystem vermittelt (Piepoli et al. 1996; Rodman et al. 2003; Scott et al. 2003; Seals und Victor 1991).

Als Folge resultieren eine erhöhte Herzfrequenz, ein Anstieg des Blutdrucks, ein verstärkter Atemantrieb und eine periphere sympathische Aktivierung (Mark et al. 1985; Mitchell et al. 1983). Bei exzessiven, wiederholten Atemanstrengungen des Diaphragmas lässt sich eine erhöhte Aktivität der afferenten Typ-IV-Fasern ableiten und mikroneurographisch ein deutlicher Anstieg der sympathischen Aktivierung nachweisen (Dempsey et al. 2002; Derchak et al. 2002; Hill 2000).

Bei COPD-Patienten leistet die Atemmuskulatur und insbesondere das Diaphragma aufgrund der inflammatorisch umgebauten Atemwege, übermäßige Atemarbeit. Im Zuge dessen entstehen diverse Verletzungen der Myozyten mit nachgewiesenem Remodeling, wodurch es zur Aktivierung des Metaboreflexes kommen könnte (Levine et al. 2003; Orozco-Levi et al. 2001; Ottenheijm et al. 2008).

Inwieweit und in welchem Ausmaß der Metaboreflex zur sympathischen Aktivierung bei COPD beiträgt ist bisher noch nicht abschließend geklärt worden.

Roseguini et al. konnten bei COPD-Patienten nach muskulärer Anstrengung des Arms, gefolgt von zirkulatorischer Okklusion einen signifikant erhöhten, aber gegenüber gesunden Kontrollpersonen relativ abgeschwächten Metaboreflex, gemessen als Gefäßwiderstand in den Unterschenkeln (CVR), feststellen (Roseguini et al. 2008). Die mikroneurographisch gemessene muskelsympathische Nervenaktivität und der Gefäßwiderstand in den Unterschenkeln korrelieren nach muskulärer 
Anstrengung sowohl intensitäts- als auch zeitabhängig eng miteinander (Seals 1989). Ähnliche Ergebnisse eines abgeschwächten Metaboreflexes konnten sowohl Sala-Mercado et al. nach Herzinsuffizienz-Induktion im Tierexperiment, als verminderte kardiale Reflexantwort messen als auch Kon et al. und Negrao et al. bei Herzinsuffizienz-Patienten beobachten, wobei das Ausmaß der Abschwächung, gemessen über den CVR und die Mikroneurographie, mit der Schwere der Herzinsuffizienz korrelierte (Kon et al. 2004; Negrao et al. 2001; Sala-Mercado et al. 2007).

Des Weiteren wurde bei gesunden Probanden durch fünfwöchiges Training der inspiratorischen Muskeln eine signifikante Abnahme der Metaboreflex-vermittelten kardiovaskulären Antwort in Form eines Herzfrequenz- und Blutdruckanstiegs bei respiratorischer Belastung im Vergleich zur Kontrollgruppe beobachtet (Witt et al. 2007).

Diese Studien legen in ihrer Gesamtheit die Hypothese nahe, dass durch kontinuierliche Stimulation, wie sie bei COPD- und Herzinsuffizienzpatienten vorliegt oder wie sie bei Gesunden simuliert werden kann, eine lokale Desensibilisierung der chemosensitiven Nervenendigungen oder der afferenten TypIII- und -IV-Nervenfasern mit konsekutiver Abschwächung der Intensität der Metaboreflexvermittelten kardiovaskulären und sympathischen Antwort stattfindet (Witt et al. 2007).

Raupach et al. konnten kürzlich zeigen, dass eine Verdopplung der Atemarbeit bei COPD-Patienten über einen kurzen Zeitraum keinen signifikanten Effekt auf die mikroneurographisch gemessene sympathische Aktivierung hat (Raupach et al. 2010).

Zusammenfassend scheint der Metaboreflex bei COPD-Patienten, insbesondere bei chronischer Aktivierung, durch eine vermeintliche Abschwächung, zumindest keine prominente Rolle in der Erhöhung der sympathischen Aktivität einzunehmen.

\subsection{Baroreflex}

Bei Patienten mit COPD konnte in verschiedenen Studien eine erniedrigte Baroreflex-Sensitivität gezeigt werden (Andreas et al. 2005). Dies gelang über spontane Variationen des Blutdrucks und der Herzfrequenz (Bartels et al. 2000), dem Phenylephrin-Test (Patakas et al. 1982) und dem ValsalvaManöver (Hjalmarsen et al. 1996). Bei Herzinsuffizienz-Patienten bewirkt eine verlangsamte Atmung dementsprechend eine Erhöhung der arteriellen Baroreflex-Sensitivität mit Erniedrigung des Blutdrucks über sympatho-inhibitorische Afferenzen (Bernardi et al. 2002). Raupach et al. konnten auch bei COPD-Patienten nachweisen, dass eine verlangsamte Atmung gegenüber der Kontrollgruppe zu einem signifikanten Abfall der mikroneurographisch gemessenen sympathischen Aktivierung führt (Raupach et al. 2008). Dies scheint unter anderem durch eine erhöhte Baroreflex-Sensitivität und/oder erniedrigte Chemoreflex-Sensitivität mit einhergehender afferenter Sympathikusinhibition bedingt zu sein (Bernardi et al. 2001). 


\subsection{Lungendehnungsreflex}

Ein weiterer, die sympathische Aktivität fördernder Faktor, könnte der Lungendehnungsreflex oder Hering-Breuer-Reflex darstellen. Bei forcierter Inspiration kommt es beim Gesunden über pulmonale vagale Afferenzen zur Hemmung respiratorischer Zentren der Medulla oblongata und zur protektiven Sympathoinhibition (Seals et al. 1990). Bei Patienten mit Herzinsuffizienz steht die sympathische Aktivierung in Verbindung mit einer abgeschwächten sympathoinhibitorischen Wirkung des Lungendehnungsreflexes (Goso et al. 2001). Daher würde diese veränderte Reflexantwort zu einer erhöhten sympathischen Aktivierung beisteuern.

Bei der Herzinsuffizienz wurde, wie oben bereits beschrieben, in verschiedenen Studien versucht, das Ausmaß der zentralen Modulation auf die Pathogenese der sympathischen Aktivierung genauer zu evaluieren. Zum jetzigen Zeitpunkt liegen bei der COPD oder Emphysemen diesbezüglich keine Studien vor.

Da sowohl bei der Herzinsuffizienz als auch bei der COPD eine systemische Entzündungsreaktion, eine Aktivierung des Renin-Angiotensin-Aldosteron-Systems und eine sympathische Aktivierung vorliegen, könnten auch bei der COPD ähnliche Mechanismen in der zentralen Modulation zur sympathischen Aktivierung beitragen.

\subsubsection{Konsequenzen der erhöhten sympathischen Aktivierung}

Vergleicht man die Herzinsuffizienz und die COPD miteinander, so lassen sich sowohl in der Pathophysiologie als auch in der klinischen Symptomatik auffallende Analogien feststellen. Die unerwünschten Begleiterscheinungen der neurohumoralen Aktivierung bei Herzinsuffizienz könnten daher auch bei der COPD relevant sein (Andreas et al. 2005).

Bei der Herzinsuffizienz nimmt die erhöhte sympathische Aktivität eine zentrale Rolle in der Pathophysiologie der Erkrankung ein und geht mit einer ungünstigen Prognose einher (Andreas et al. 2005; Brouwer et al. 1996; Cohn et al. 1984). Die mikroneurographisch gemessene sympathische Aktivität korreliert dabei mit dem funktionellen Grad der Herzinsuffizienz nach der New York Heart Association (NYHA) und ist ein prognostischer Indikator in Bezug auf die Mortalität (Barretto et al. 2009; Munhoz et al. 2009).

Die sympathische Aktivierung bei Herzinsuffizienz ist assoziiert mit gesteigerter Atemfrequenz, vermehrter Totraumventilation und Dyspnoe bei Belastung (Coats et al. 1992; Gosker et al. 2003). Weiterhin gibt es eine starke Assoziation zum Vorliegen einer Kachexie, die unabhängig vom Grad der Herzinsuffizienz mit einer hohen Mortalität einhergeht und bei der das Ausmaß stark mit der sympathischen Aktivierung und immunologischen Faktoren korreliert (Anker und Coats 1999; Anker 
und Sharma 2002). Pathophysiologisch führt die sympathische Aktivierung im Zusammenspiel mit der systemischen Entzündungsreaktion zu einer endothelialen Dysfunktion mit erniedrigter Vasodilatation in den Skelettmuskeln während einer Belastung. Aus der erhöhten Nachlast resultiert eine verstärkte kardiale Anstrengung, die prädisponierend für eine Ischämie und ventrikuläre Arhythmien ist und daher die Wahrscheinlichkeit für signifikante kardiale Ereignisse erhöht. Des Weiteren begünstigt eine erhöhte sympathische Aktivierung eine Verringerung von ausdauernden Typ-I-Muskelfasern, Kardiomyozytenverletzungen und -apoptose, Muskelatrophie und Lipolyse (Anker und Coats 1999; Barretto et al. 2009; Katz et al. 2005; Negrao et al. 2001; Olivetti et al. 1997; Packer 1992).

Den erhöhten Katecholaminspiegeln infolge der sympathischen Aktivierung kommt bei der Herzinsuffizienz eine besondere Bedeutung zu. Die Instillation von Noradrenalin in Kardiomyozytengewebe führt, als Folge einer gesteigerten Sarkolemmpermeabilität, einer cAMPvermittelten Kalziumüberladung der Zelle und der Entstehung oxidativer Katecholaminmetabolite, zu toxischen Effekten, welche mit der Abnahme der zellulären Aktivität und Lebensfähigkeit einhergehen (Mann et al. 1992; Todd et al. 1985). Im Tiermodell konnte demonstriert werden, dass eine permanente Katecholamingabe die Apoptose über den reaktiven Sauerstoffspezies-TNF-CaspaseSignalweg induziert. Außerdem schädigen reaktive Zwischenprodukte, unabhängig von Adrenorezeptoren, direkt die Kardiomyozyten. Dieser Schritt ist daher nicht durch eine Blockade der Betarezeptoren zu beeinflussen (Communal et al. 1998; Fu et al. 2004; Neri et al. 2007). Inwieweit ein toxischer Effekt der Katecholamine schädigend auf die Skelettmuskeln, insbesondere das Diaphragma, wirkt, wurde bisher noch nicht genau geklärt.

Nicht nur im Tierexperiment, sondern auch bei Herzinsuffizienz-Patienten gibt es deutliche Anzeichen, dass chronisch erhöhte Katecholaminspiegel eine Abnahme und Desensibilisierung von $\alpha$ und $\beta$-Adrenorezeptoren mit konsekutiver Reduzierung der Empfindlichkeit auf sympathische Stimuli bewirken (Bristow et al. 1982; Engelhardt et al. 1996; Feng et al. 1999; Nishikawa et al. 1994; Roseguini et al. 2008; Seals und Dinenno 2004). Daher könnten die chronisch erhöhten Katecholaminspiegel, bedingt durch die sympathische Aktivierung, bei COPD-Patienten über eine Desensibilisierung der bronchodilatatorisch wirkenden $\beta$-Adrenorezeptoren zur verstärkten Bronchokonstriktion und zu weiteren funktionalen Einschränkungen beitragen (Borst et al. 1999; Weiss und Shore 2004).

Bei der COPD steht der Grad der Skelettmuskeldysfunktion in enger Beziehung zur klinischen Symptomatik und Prognose (Mador 2002). Da die erhöhte sympathische Aktivierung neben der systemischen Entzündungsreaktion als Ursache und verschlechternder Faktor der Skelettmuskeldysfunktion zu sehen ist, könnte dies auch bei COPD-Patienten der Fall sein (Andreas et al. 2005; Hofford et al. 1990). 
Wie Herzinsuffizienz-Patienten leiden auch COPD-Patienten häufig unter einer die Prognose verschlechternden Kachexie (Wagner 2008). Diese ist dabei nicht nur auf einen erhöhten Energieumsatz und eine Inaktivitätsatrophie zurückzuführen, sondern korreliert eng mit der gesteigerten systemischen Entzündungsreaktion und dem zirkulierenden Leptinspiegel (Debigare et al. 2001; Koehler et al. 2007; Schols et al. 1999; Sergi et al. 2006).

Leptin hat neben der Wirkung auf die Nahrungsaufnahme, den Lipidmetabolismus und die Thermoregulation weitere wichtige immunologische Effekte. Es ist als Zytokin involviert in die Rekrutierung, Aktivierung und das Überleben von diversen inflammatorischen Entzündungszellen, wobei es invers mit deren Apoptose korreliert. Bei der COPD ist Leptin im Sputum und der bronchialen Submukosa signifikant erhöht und korreliert mit der lokalen Konzentration von TNF- $\alpha$ und CRP. (Broekhuizen et al. 2005; Bruno et al. 2005b; Calikoglu et al. 2004; Fantuzzi und Faggioni 2000).

Insbesondere die Wirkung auf die neutrophilen Granulozyten, die eine prominente Rolle in der Pathophysiologie der COPD einnehmen, könnte diesbezüglich bedeutend sein. Im Speziellen ist Leptin in der Lage der Apoptose von neutrophilen Granulozyten entgegenzuwirken und diese, unter anderem über einen fördernden Effekt auf die TNF- $\alpha$-Expression, zu aktivieren (Bruno et al. 2005a; Caldefie-Chezet et al. 2001; Zarkesh-Esfahani et al. 2004). Da die Aktivierung des autonomen Nervensystems möglicherweise die zirkulierenden Leptinspiegel bei COPD-Patienten beeinflusst und dies offenbar mit einer lokal verstärkten inflammatorischen Antwort einhergeht, käme dem Leptin sowohl als metabolischer wie auch immunologischer Faktor eine Bedeutung in der Pathophysiologie der COPD zu (Malli et al. 2010; Snitker et al. 1997; Takabatake et al. 2001).

Eine weitere Auswirkung der sympathischen Aktivierung stellt die Peroxidation von Lipiden dar, in deren Folge es zu einer erschwerten Gewichtszunahme und einer Steigerung der Morbidität kommt (Snitker et al. 1998).

Eine Aktivierung des sympathischen Nervensystems wirkt stimulierend auf die Reninsekretion und treibt so das Renin-Angiotensin-Aldosteron-System an (Goldsmith 2004).

In verschiedenen Studien konnte gezeigt werden, dass eine verstärkte Aktivierung des ReninAngiotensin-Aldosteron-Systems sowohl bei der COPD als auch bei der Herzinsuffizienz mit einer Reihe unerwünschter Konsequenzen einhergeht. So ließen sich erhöhte Plasmarenin-, Plasmaaldosteron- und Angiotensin-II-Werte feststellen und eine Assoziation mit einer Natriumretention und peripheren Ödemen (Francis GS 1989; Stewart et al. 1994). Im Tierexperiment und bei Patienten mit Herzinsuffizienz konnte zudem nachgewiesen werden, dass es zur erhöhten Expression von Angiotensinrezeptoren im Myokard kommt, über die das pathologische Remodeling 
forciert wird (Adams 2004; Kaprielian et al. 1997; Lambert et al. 1995; Nio et al. 1995; Suzuki et al. 1993).

Eine vergleichbare Rolle könnten die in hoher Dichte im Lungengewebe exprimierten $\mathrm{AT}_{1^{-}}$Rezeptoren bei der COPD einnehmen, wo sie anscheinend Auswirkungen auf die Apoptose der Epithelzellen und das Wachstum von Fibroblasten haben (Llorens-Cortes et al. 1994; Molteni et al. 2003; Wang R et al. 1999). Angiotensin II könnte daher ein entscheidender Mediator für einen Lungenschaden sein und einen emphysematösen Umbau begünstigen (Wosten-van Asperen et al. 2008).

In der Therapie der Herzinsuffizienz werden seit langem Medikamente eingesetzt, deren Wirkung darauf abzielt, die neurohumorale Aktivierung zu reduzieren und dem pathologischen Remodeling entgegenzuwirken. Darunter fallen Angiotensin-Rezeptor-Antagonisten, $\beta$-Blocker, AldosteronAntagonisten und ACE-Hemmer, welche effektiv die Lebensqualität verbessern und die Morbidität und Mortalität verringern (Jessup und Brozena 2003).

Der Effekt der $\beta$-Blocker in Bezug auf das sympathische Nervensystem wird unter anderem über eine Hemmung des katecholaminergen kardiotoxischen Effektes, eine Schwächung der neurohumoralen Vasokonstriktion, der Wachstumsförderung und von pro-apoptotischen Systemen, wie beispielsweise dem RAAS, vermittelt und wirkt somit dem kardiomyozytären Remodeling entgegen (Adamson und Gilbert 2006; Triposkiadis et al. 2009).

Die Anwendung von Angiotensin-II-Typ-1-Rezeptor-Blockern hemmt sowohl die Aktivität des sympathischen als auch des Renin-Angiotensin-Aldosteron-Systems (Esler 2002; Kawamura et al. 2009) und könnte daher ähnlich wie bei der Herzinsuffizienz atrophischen Prozessen von Skelett- und respiratorischer Muskulatur entgegenwirken.

Kawamura et al. konnten bei Patienten mit Linksherzinsuffizienz zeigen, dass eine Kombination aus ACE-Hemmer und $\mathrm{AT}_{\mathrm{II}}$-Rezeptor-Blockern die sympathische Aktivierung, gemessen über die Mikroneurographie, signifikant erniedrigte und die arterielle und kardiale Baroreflex-Sensitivität signifikant erhöhte (Kawamura et al. 2009).

In Ratten mit Rechtsherzinsuffizienz schützte die Applikation des $\mathrm{AT}_{\mathrm{II}}$-Rezeptor-Blocker Irbesartan vor der Entwicklung einer Apoptose-abhängigen Skelettmuskelatrophie (Dalla Libera et al. 2001). Darüber hinaus wurde bereits gezeigt, dass die Langzeittherapie mit dem ACE-Hemmer Perindopril bei Patienten mit Linksherzinsuffizienz die respiratorische Muskelkraft signifikant erhöhte (Coirault et al. 2001). Bisher konnte bei COPD-Patienten durch die Therapie mit Irbesarten keine signifikante Verstärkung der respiratorischen Muskelkraft nachgewiesen werden (Andreas et al. 2006). Allerdings konnte im Maus-Modell, in Elastase-induzierten Emphysemen, positive Wirkungen der Behandlung mit dem Angiotensinrezeptorblocker Irbesartan in Bezug auf die Emphysemschwere, die Lungenbiomechanik und die klinische Belastbarkeit gegenüber der Kontrollgruppe gefunden werden (Raupach et al. 2011). 
Da eine permanent erhöhte sympathische Aktivierung zur Vasokonstriktion, insbesondere von pulmonal venösen Gefäßen führt, könnte dies zur Entwicklung einer pulmonalen Hypertonie beitragen und somit die Morbidität der Erkrankung weiter verstärken (Dauber und Weil 1983; Duplain et al. 1999; Nishikawa et al. 1994).

\subsection{Zielsetzung der Arbeit}

Sowohl bei der chronischen Herzinsuffizienz als auch bei der chronisch obstruktiven Lungenerkrankung handelt es sich um medizinisch, wie ökonomisch schwerwiegende Erkrankungen, die die Lebensqualität und das Überleben der betroffenen Patienten in beträchtlichem Maße einschränkt. Die nachweislich erhöhte sympathische Aktivierung korreliert bei der Herzinsuffizienz mit einer signifikant schlechteren Prognose. Eine medikamentöse Therapie, die den Sympathikotonus mindert, führt dabei zu einer signifikanten Verbesserung der Prognose (Andreas et al. 2005; Barretto et al. 2009; Munhoz et al. 2009).

Da es bei der COPD bisher keine vergleichbaren Untersuchungen gibt, war es Ziel der Arbeit, retrospektiv zu untersuchen, ob der Grad der sympathischen Aktivierung Einfluss auf Morbidität und Mortalität von COPD-Patienten hat. Zudem wurden die Ergebnisse mit einer gesunden Kontrollgruppe und Herzinsuffizienz-Patienten verglichen.

Um dies zu überprüfen, wurden folgende Fragestellungen untersucht:

1. Gibt es einen signifikanten Unterschied im Grad der gemessenen sympathischen Aktivierung zwischen bereits verstorbenen Patienten gegenüber lebenden Patienten mit der gleichen Erkrankung?

2. Hatten Patienten, die seit der mikroneurographischen Untersuchung aufgrund ihrer Erkrankung stationär im Krankenhaus behandelt werden mussten, eine signifikant erhöhte sympathische Aktivierung gegenüber Patienten, die nicht stationär behandelt werden mussten?

3. Korreliert der Grad der sympathischen Aktivierung mit der Anzahl an Krankenhausaufenthalten?

4. Haben Patienten, die entweder im Krankenhaus behandelt werden mussten oder verstorben sind, einen signifikant erhöhten Grad der sympathischen Aktivierung gegenüber Patienten, die weder verstorben sind, noch stationär behandelt werden mussten? 
Als klinische Endpunkte dieser Studie werden festgesetzt:

1. Das Vorliegen eines oder mehrerer Krankenhausaufenthalte im Follow-Up aufgrund einer Exazerbation der jeweiligen Grunderkrankung als valider Parameter zur Evaluation der krankheitsassoziierten Morbidität

2. Der Tod des Patienten im Follow-Up als Parameter zur Evaluation der Mortalität

3. Das Vorliegen mindestens eines Krankenhausaufenthalts oder des Todes des Patienten im Follow-Up als klinisch relevanter und biologisch plausibler zusammengesetzter Endpunkt. 


\section{METHODIK}

\subsection{Probandencharakteristika}

\subsubsection{Herzinsuffizienz-Patienten}

Das Kollektiv der Herzinsuffizienz-Patienten erfüllte zum Zeitpunkt der Untersuchungen folgende Kriterien und Charakteristika:

Einschlusskriterien:

- Chronische Herzinsuffizienz mit funktionaler Beeinträchtigung nach NYHA Grad II-III (Hunt et al. 2005) aufgrund einer koronaren Herzerkrankung oder idiopathischen Kardiomyopathie

- Linksventrikuläre Ejektionsfraktion $\leq 35 \%$.

Ausschlusskriterien:

- Myokardinfarkt

- $\quad$ Lungenödem in den letzten 6 Monaten vor Untersuchung

- Obstruktive Schlafapnoe

- Nieren-, Leber-, oder Lungenerkrankung ( $\mathrm{FEV}_{1}<70 \%$ des Solls)

- Polyneuropathie und andere neurologische Erkrankungen

- Sympathomimetische Medikation (Theophylline, Moxonidin, Clonidin, $\beta 2$-Sympathomimetika). 


\subsubsection{COPD-Patienten}

Das Kollektiv der COPD-Patienten erfüllte zum Zeitpunkt der Untersuchung folgende Kriterien:

Einschlusskriterien:

- COPD Grad II-IV mit äquivalenter Therapie nach GOLD-Schema (Rabe et al. 2007)

- Alter: 30-80 Jahre

- Stabiler Sinusrhythmus

- Keine Exazerbation der COPD innerhalb der letzten 3 Monate vor Untersuchung.

Ausschlusskriterien:

- Langzeit-Sauerstoff-Applikation

- Obstruktive Schlafapnoe

- Chronische Herzinsuffizienz

- Myokardinfarkt

- Systemische sympathomimetische Medikation (Theophyllin, Moxonidin, Clonidin, orale $\beta 2$-Sympathomimetika)

- Erkrankungen mit sympathischer Aktivierung

- Insulin-pflichtiger Diabetes mellitus

- Klinisch nachgewiesene Polyneuropathie

- Einnahme von Diuretika vor der Untersuchung.

\subsubsection{Kontrollpersonen}

Die Kontrollpersonen wurden in die Studie miteinbezogen, wenn sie unter keiner bekannten chronischen Erkrankung litten und keine regelmäßige Medikation bekamen. Die Kontrollpersonen waren in den jeweiligen Studien zu den Patienten 1:1 hinsichtlich Geschlechtes, Alter und Gewicht gematcht. 


\subsection{Studienbeschreibung}

\subsubsection{Studiendesign}

In einer Nachbeobachtungsstudie wurden bereits mikroneurographisch untersuchte Patienten und Kontrollpersonen aus vier vorhergehenden Studien der Universitätsmedizin Göttingen befragt. Es handelte sich dabei um 20 COPD-Patienten, 20 Herzinsuffizienz-Patienten und 23 Kontrollpersonen. Die COPD-Patienten rekrutieren sich aus zwei verschiedenen Studien, wobei 6 Patienten im Zeitraum von Oktober bis Dezember 1998 und 14 Patienten zwischen Oktober 2005 und Februar 2006 untersucht wurden (Heindl et al. 2001b; Raupach et al. 2008).

Die Herzinsuffizienz-Patienten wurden zwischen Juni 2000 und Oktober 2001 untersucht (Andreas et al. 2004; Raupach et al. 2008).

Die Kontrollpersonen rekrutieren sich aus allen vier verschiedenen Studien und wurden im Zeitraum vom Mai 1998 bis Februar 2006 untersucht (Heindl et al. 2001a).

Von den beiden Patientenkollektiven konnten jeweils alle 20 Patienten kontaktiert beziehungsweise das Todesdatum ermittelt werden. Von den ursprünglich 40 Kontrollpersonen lagen nur bei 28 Kontrollpersonen mikroneurographische Daten vor, von denen 23 letztendlich in die Studie miteinbezogen werden konnten.

Die Studie wurde von der Ethikkommission der Medizinischen Fakultät der Universität Göttingen begutachtet. Dem Antrag (Nr. 22/9/10) wurde am 3.12.2010 stattgegeben. Das Prüfzentrum war das Universitätsklinikum Göttingen in Kooperation mit der Lungenfachklinik Immenhausen.

\subsubsection{Studienprotokoll}

Initial wurde über das Einwohnermeldeamt geklärt, ob und wann der jeweilige Proband verstorben war. Wenn der Todesfall nicht eingetreten war, wurde der Proband zunächst schriftlich kontaktiert. In dem in der Anlage beigefügten Brief fand die ausführliche Aufklärung des Probanden über Art, Umfang und Zweck der Studie statt. Darüber hinaus wurde der Proband über die gesetzlichen Datenschutzbestimmungen aufgeklärt. Im Anschreiben wurden außerdem im Voraus die Fragen und die zu unterschreibende Einwilligungserklärung beigefügt. Probanden, die die Einwilligungserklärung unterschrieben zurückgesandt hatten, wurden telefonisch kontaktiert.

Falls die Probanden keine weiteren Fragen oder Informationsbedarf hatten, wurden Sie persönlich, entsprechend dem Fragenkatalog, standardisiert befragt. Die Patienten wurden dabei gefragt, ob sie seit der mikroneurographischen Untersuchung aufgrund ihrer jeweiligen Erkrankung (COPD oder Herzinsuffizienz) stationär behandelt werden mussten. Die Kontrollpersonen wurden gefragt, ob sie aufgrund einer Herz- oder Lungenerkrankung stationär behandelt werden mussten. 
Die Patienten und Probanden wurden für die Teilnahme an der Studie weder finanziell noch anderweitig entschädigt.

Von den bereits verstorbenen Probanden wurden etwaige Krankenhausaufenthalte nicht erfasst.

\subsection{Messmethoden}

\subsubsection{Mikroneurographische Messung}

Die Mikroneurographie stellt eine valide und reproduzierbare Methode zur Evaluation der sympathischen Aktivität dar und zeigt über einen Zeitraum von vielen Jahren eine hohe intraindividuelle Konstanz. Sie ist dabei anderen Methoden, wie der Bestimmung der Herzfrequenzvariabilität oder der Katecholaminespiegel im Serum oder Urin, überlegen (Grassi et al. 2009; Jennings 1998; Lahiri et al. 2008; Vallbo et al. 2004).

In allen drei Studien wurde die mikroneurographische Untersuchung in gleicher Weise, wie folgend beschrieben, in der Universitätsmedizin Göttingen durchgeführt.

In leicht angewinkelter, stabiler Lagerung des Beines wurde der Nervus peroneus palpatorisch und mittels transkutaner Elektrostimulation (1-5mA, 0.1-0.3 ms, 1Hz) oberflächlich lokalisiert. Anschließend wurde eine dünne, isolierte Wolframelektrode (Durchmesser der Spitze ca. 1-15 $\mu \mathrm{m}$, Durchmesser des Schafts ca. $200 \mu \mathrm{m}$ ) als Referenznadel 1-2 cm vom Nerven entfernt subkutan platziert. Mittels einer zweiten Wolframelektrode wurde dann versucht den motorischen Anteil des Nerven unter Elektrostimulation zu punktieren. Daraufhin wurden die sympathischen Efferenzen zu den Gefäßen der Wadenmuskulatur dargestellt. Um einer Verfälschung der Ergebnisse durch übermäßige Manipulation vorzubeugen, wurde die Suche des Signals auf maximal eine Stunde und drei transkutane Punktionsversuche begrenzt.

Der Grad der sympathischen Aktivierung wurde als Ausschläge pro Minute (b/min) und als Ausschläge pro 100 Herzschläge (b/100hbs) gemessen. 


\subsubsection{Sonstige Messmethoden}

Bei den Patienten und Probanden wurden während der vorhergehenden Studien neben der Mikroneurographie folgende Messungen durchgeführt, die in dieser Studie statistisch verwertet wurden:

- standardisierte 12-Kanal EKG Ableitung

- Lungenfunktionsuntersuchung

- arterielle Blutgasanalyse

- Echokardiographie.

Die Messungen wurden in allen drei Studien auf standardisierte Weise durchgeführt (Andreas et al. 2004; Heindl et al. 2001b; Raupach et al. 2008).

\subsection{Statistische Auswertung}

Die statistische Auswertung wurde mit dem Programm „Statistica 10“ für Microsoft Windows in Zusammenarbeit mit der Abteilung der Medizinischen Statistik der Universitätsmedizin Göttingen durchgeführt. Dabei wurden die unterschiedlichen Gruppen mithilfe des zweiseitigen Student's t-Tests für unabhängige Variablen hinsichtlich der erfassten Parameter verglichen. Ferner wurden eine Überlebenszeitanalyse mithilfe des Kaplan-Meier-Schätzers und dem log-rank-Test durchgeführt und die erfassten Parameter untereinander miteinander korreliert. Eine statistische Signifikanz wurde bei einem zweiseitigen p-Wert $<0,05$ angenommen. 


\section{ERGEBNISSE}

\section{1 Allgemeine statistische Charakteristika der Probandengruppen}

Von den zu kontaktierenden Probanden in der Gruppe der COPD- und Herzinsuffizienzpatienten konnten jeweils alle 20 Teilnehmer, bei den gesunden Kontrollpersonen konnten 23 von 28 zu kontaktierenden Personen letztendlich in die Studie einbezogen werden. Da nicht alle ursprünglichen Kontrollpersonen in die Studie miteinbezogen werden konnten, war das 1:1 Matching der zugrundeliegenden Studien nicht mehr gegeben.

\subsubsection{Herzinsuffizienz-Patienten versus Kontrollgruppe}

Die Herzinsuffizienz-Patienten hatten gegenüber der Kontrollgruppe eine signifikant schlechtere Lungenfunktion (gemessen als $\mathrm{FEV}_{1}$-Ist/FEV ${ }_{1}$-Soll) und eine erhöhte periphere sympathische Aktivierung, gemessen in $\mathrm{b} / \mathrm{min}(\mathrm{p}=0,0496)$ und $\mathrm{b} / 100 \mathrm{hbs}(\mathrm{p}=0,0496$; siehe Tab. 2). Zum Zeitpunkt der mikroneurographischen Untersuchung erhielten die insgesamt 20 Herzinsuffizienz-Patienten folgende Medikation:

o ACE-Hemmer: 16 Patienten

o $\beta$-Blocker: 13 Patienten

o $\quad$ AT $_{\text {II }}$-Blocker: 1 Patient

o Diuretika: 11 Patienten

o Digitalisglykoside: 9 Patienten. 
Tabelle 2: Vergleich allgemeiner Charakteristika der Herzinsuffizienz-Patienten gegenüber der Kontrollgruppe (Mittelwert \pm Standardabweichung)

\begin{tabular}{|c|c|c|c|}
\hline & $\begin{array}{c}\text { Herzinsuffizienz - } \\
\text { Patienten }\end{array}$ & Kontrollgruppe & p-Wert (2-seitig) \\
\hline Anzahl (n) & 20 & 23 & $0,013^{*}$ \\
\hline Geschlecht (m/w) & $19 / 1$ & $15 / 8$ & $0,02^{*}$ \\
\hline $\begin{array}{c}\text { Mittleres Follow-Up } \\
\text { (Monate) }\end{array}$ & $116,2 \pm 6,3$ & $93,2 \pm 42,3$ & $0,039^{*}$ \\
\hline Alter (Jahren) & $54,3 \pm 11,8$ & $60,7 \pm 6,1$ & 0,076 \\
\hline Körpergröße (cm) & $176 \pm 5$ & $172 \pm 9$ & 0,11 \\
\hline Gewicht (kg) & $81,0 \pm 10,2$ & $75,4 \pm 11,9$ & 0,53 \\
\hline Body-Mass-Index & $26,2 \pm 2,9$ & $25,6 \pm 3,4$ & $0,0013^{*}$ \\
\hline $\begin{array}{c}\text { FEV } \mathbf{1}_{\mathbf{1}} \text {-Ist/FEV } \mathbf{1} \text {-Soll (in } \\
\text { \% der Norm) }\end{array}$ & $90 \pm 20$ & $110 \pm 10$ & 0,34 \\
\hline $\begin{array}{c}\text { Herzfrequenz } \\
\text { (Schläge/min) }\end{array}$ & $67,2 \pm 10,5$ & $63,8 \pm 7,6$ & $0,0496^{*}$ \\
\hline MSNA (b/min) & $37,7 \pm 15,3$ & $29,4 \pm 10,0$ & $0,0496^{*}$ \\
\hline MSNA (b/100hbs) & $57,0 \pm 24,8$ & $43,9 \pm 15,2$ & \\
\hline
\end{tabular}




\subsubsection{COPD-Patienten versus Kontrollgruppe}

Die COPD-Patienten hatten gegenüber der Kontrollgruppe eine signifikant schlechtere Lungenfunktion (gemessen als $\mathrm{FEV}_{1}-\mathrm{Ist}_{\mathrm{FEV}}$-Soll), schlechtere Blutgas-Werte (pO2 und pCO2), eine schnellere Herzfrequenz und eine höhere periphere sympathische Aktivität sowohl in b/min $(\mathrm{p}=0,000041)$ als auch $\mathrm{b} / 100 \mathrm{hbs}(\mathrm{p}=0,00034$; siehe Tab. 3$)$.

In Bezug auf Geschlecht, Alter, Körpergröße, Gewicht und Body-Mass-Index bestand kein signifikanter Unterschied zwischen den Gruppen (siehe Tab. 3).

Tabelle 3: Vergleich allgemeiner Charakteristika der COPD-Patienten gegenüber der Kontrollgruppe (Mittelwert \pm Standardabweichung)

\begin{tabular}{|c|c|c|c|}
\hline & COPD -Patienten & Kontrollgruppe & p-Wert (2-seitig) \\
\hline Anzahl(n) & 20 & 23 & 0,49 \\
\hline Geschlecht (m/w) & $15 / 5$ & $15 / 8$ & 0,42 \\
\hline $\begin{array}{c}\text { Mittleres Follow-up } \\
\text { (Monate) }\end{array}$ & $83,6 \pm 36,0$ & $93,2 \pm 42,3$ & 0,55 \\
\hline Alter (Jahren) & $62 \pm 7,6$ & $60,7 \pm 6,1$ & 0,78 \\
\hline Körpergröße (cm) & $171 \pm 9,2$ & $172 \pm 9$ & 0,46 \\
\hline Gewicht (kg) & $78,5 \pm 14,5$ & $75,4 \pm 11,9$ & 0,31 \\
\hline Body-Mass-Index & $26,9 \pm 4,9$ & $25,6 \pm 3,4$ & $0,000001^{*}$ \\
\hline $\begin{array}{c}\text { FEV } \mathbf{1} \text {-Ist/FEV } \mathbf{1} \text {-Soll } \\
\text { (in \% der Norm) }\end{array}$ & $50 \pm 20$ & $110 \pm 10$ & $0,00011^{*}$ \\
\hline pO2 (art. in mmHg) & $72,7 \pm 9,9$ & $85,2 \pm 8,2$ & $0,021^{*}$ \\
\hline pCO2 (art. in mmHg) & $41,9 \pm 3,8$ & $39,1 \pm 3,5$ & $0,00023^{*}$ \\
\hline $\begin{array}{c}\text { Herzfrequenz } \\
\text { (Schläge/min) }\end{array}$ & $83,7 \pm 17,6$ & $63,8 \pm 7,6$ & $0,000041^{*}$ \\
\hline MSNA (b/min) & $52,4 \pm 18,9$ & $29,4 \pm 10,0$ & $0,00034^{*}$ \\
\hline MSNA(b/100hbs) & $62,8 \pm 16,1$ & $43,9 \pm 15,2$ & \\
\hline
\end{tabular}




\subsubsection{COPD-Patienten versus Herzinsuffizienz-Patienten}

Die COPD-Patienten hatten zum Zeitpunkt der Untersuchung gegenüber den HerzinsuffizienzPatienten eine signifikant erhöhte sympathische Aktivierung in $\mathrm{b} / \mathrm{min}$ ( $\mathrm{p}=0,01$; siehe Tab 4.).

Tabelle 4: Vergleich allgemeiner Charakteristika der COPD-Patienten gegenüber HerzinsuffizienzPatienten (Mittelwert \pm Standardabweichung)

\begin{tabular}{|c|c|c|c|}
\hline & COPD-Patienten & $\begin{array}{c}\text { Herzinsuffizienz - } \\
\text { Patienten }\end{array}$ & p-Wert (2-seitig) \\
\hline Anzahl (n) & 20 & 20 & 0,083 \\
\hline Geschlecht (m/w) & $15 / 5$ & $19 / 1$ & $0,0007^{*}$ \\
\hline $\begin{array}{c}\text { Mittleres Follow-up } \\
\text { (Monate) }\end{array}$ & $83,6 \pm 36,0$ & $116,2 \pm 6,3$ & $0,021^{*}$ \\
\hline Alter (Jahren) & $62 \pm 7,6$ & $54,3 \pm 11,8$ & $0,049^{*}$ \\
\hline Körpergröße (cm) & $171 \pm 9,2$ & $176 \pm 5$ & 0,53 \\
\hline Gewicht (kg) & $78,5 \pm 14,5$ & $81,0 \pm 10,2$ & 0,57 \\
\hline Body-Mass-Index & $26,9 \pm 4,9$ & $26,2 \pm 2,9$ & $<0,000001^{*}$ \\
\hline $\begin{array}{c}\text { FEV } \text {-Ist/FEV } \mathbf{1} \text {-Soll } \\
\text { (in \% der Norm) }\end{array}$ & $50 \pm 20$ & $90 \pm 20$ & $0,0011^{*}$ \\
\hline $\begin{array}{c}\text { Herzfrequenz } \\
\text { (Schläge/min) }\end{array}$ & $83,7 \pm 17,6$ & $67,2 \pm 10,5$ & $0,01^{*}$ \\
\hline MSNA (b/min) & $52,4 \pm 18,9$ & $37,7 \pm 15,3$ & 0,39 \\
\hline MSNA (b/100hbs) & $62,8 \pm 16,1$ & $57,0 \pm 24,8$ & \\
\hline
\end{tabular}




\subsection{Spezifische statistische Ergebnisse}

\subsubsection{Herzinsuffizienz-Patienten}

\subsubsection{Vergleich der Herzinsuffizienz-Patienten mit der Kontrollgruppe}

Zum Zeitpunkt der Befragung waren 7 von 20 Herzinsuffizienz-Patienten verstorben. Bei 5 von 7 der verstorbenen Patienten konnte ein akzidentieller Tod ausgeschlossen werden. 11 von 13 befragten Patienten mussten aufgrund ihrer Erkrankung stationär behandelt werden. Bei den HerzinsuffizienzPatienten gab es gegenüber der Kontrollgruppe signifikant mehr Todesfälle ( 7 zu 1; p=0,015), mehr Probanden mit Krankenhausaufenthalten (11 zu 1; p=0,000002) und ein erhöhtes Mortalitätsrisiko mit einer kürzeren Überlebenszeit ( $\mathrm{p}=0,008$; siehe Abb. 1).

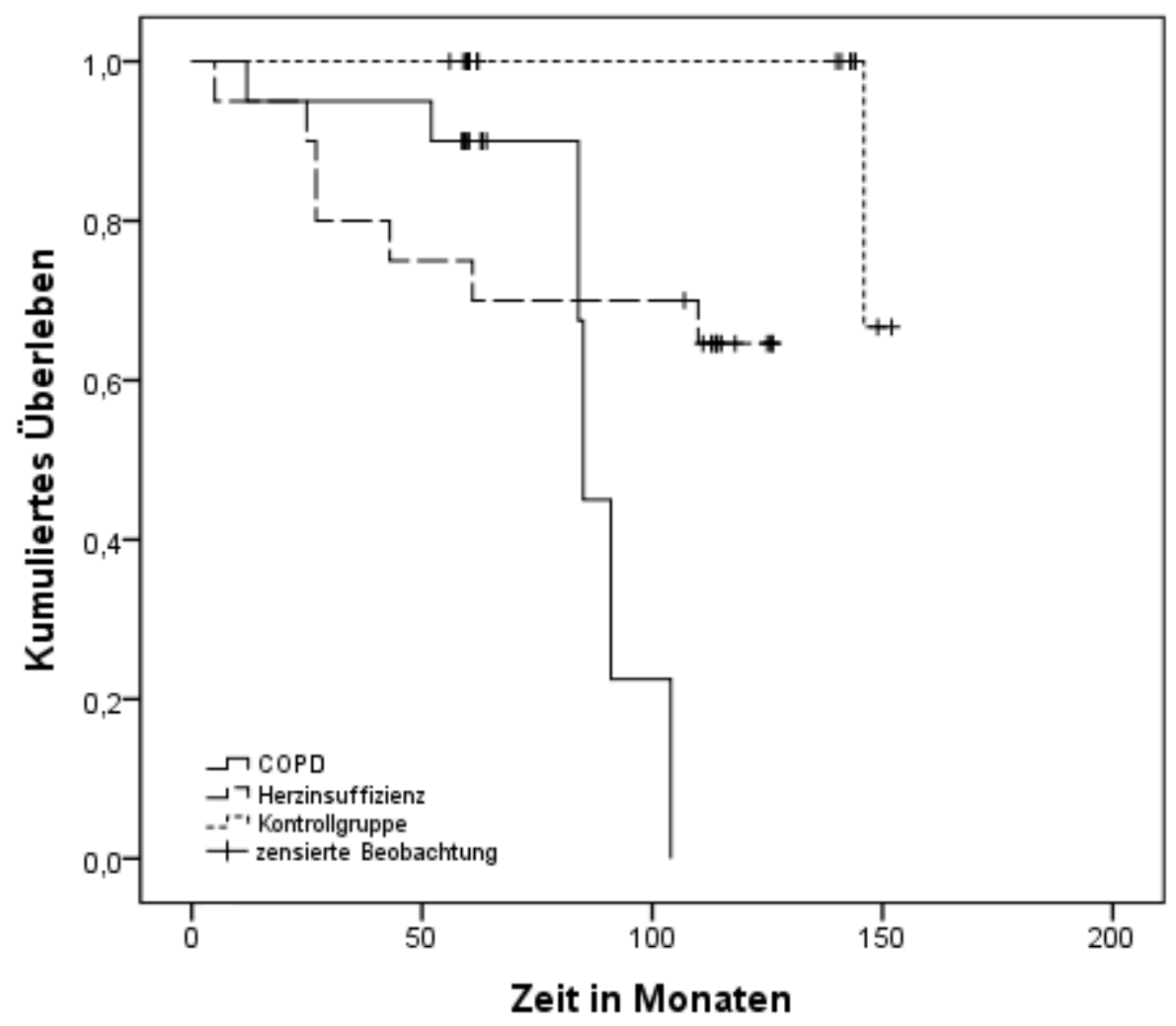

Abbildung 1: Vergleich des kumulativen Überlebens (Kaplan-Meier) von COPD-Patienten, Herzinsuffizienz-Patienten und der Kontrollgruppe 


\subsubsection{Vergleich der Herzinsuffizienz-Patienten hinsichtlich klinischer Endpunkte}

Das Ausmaß der sympathischen Aktivierung war bei verstorbenen Herzinsuffizienz-Patienten, gemessen als b/min, signifikant niedriger als bei lebenden Patienten (Mittelwert 28,1 \pm 13,1 vs 42,8 \pm 14,3; p=0,038; siehe Tab. 5).

Die mittlere Überlebenszeit und das Mortalitätsrisiko zwischen den Herzinsuffizienz-Patienten, deren sympathische Aktivierung über beziehungsweise unter dem Median liegt, unterscheidet sich im Kaplan-Meier-Schätzer nicht signifikant voneinander (b/min p=0,19).

Bei den Herzinsuffizienz-Patienten mussten 11 von 13 der lebenden Patienten mindestens einmal stationär behandelt werden. Die stationär behandelten Patienten hatten eine signifikant erhöhte sympathische Aktivität, sowohl gemessen als b/min (45,5 $\pm 13,9$ vs $28 \pm 3,4$; p=0,0062) als auch b/100hbs (68,6 $\pm 23,2$ vs 34,8 \pm 7,1; $\mathrm{p}=0,007$; siehe Tab. 5).

Die Anzahl an Krankenhausaufenthalten reichten von einem bis zu 12 (Median=2) und korrelierten nicht mit dem Ausmaß der sympathischen Aktivierung in $b / \min (p=0,92)$ oder $b / 100 h b s(p=0,89)$.

Die Patienten, die entweder einen Krankenhausaufenthalt hatten oder verstorben sind $(n=18)$ haben gegenüber den Patienten, die keines der beiden Ereignisse hatten $(n=2)$, eine signifikant erhöhte sympathische Aktivierung in $\mathrm{b} / \mathrm{min}(38,7 \pm 15,8$ vs $28 \pm 3,4$; $\mathrm{p}=0,039)$ und $\mathrm{b} / 100 \mathrm{hbs}(59,5 \pm 24,9$ vs 34,8 \pm 7,1; $\mathrm{p}=0,023$; siehe Tab. 5, Abb. 2).

Tabelle 5: Vergleich der sympathischen Aktivierung der Herzinsuffizienz-Patienten aufgeteilt nach klinischen Endpunkten

\begin{tabular}{|c|c|c|c|c|c|c|c|}
\hline & & & Anzahl (n) & $\mathbf{b} / \mathbf{m i n}$ & p-Wert & b/100hbs & p-Wert \\
\hline \multirow{6}{*}{$\begin{array}{l}\text { Klinischer } \\
\text { Endpunkt }\end{array}$} & \multirow{2}{*}{ Tod } & ja & 7 & $28,1 \pm 13,1$ & \multirow{2}{*}{$0,038 *$} & $45,1 \pm 21,5$ & \multirow{2}{*}{0,11} \\
\hline & & nein & 13 & $42,8 \pm 14,3$ & & $63,4 \pm 24,8$ & \\
\hline & \multirow{2}{*}{$\begin{array}{l}\text { Stationärer } \\
\text { Aufenthalt }\end{array}$} & ja & 11 & $45,5 \pm 13,9$ & \multirow{2}{*}{$0,0062 *$} & $68,6 \pm 23,2$ & \multirow{2}{*}{$0,007^{*}$} \\
\hline & & nein & 2 & $28,0 \pm 3,4$ & & $34,8 \pm 7,1$ & \\
\hline & \multirow{2}{*}{$\begin{array}{c}\text { Tod oder } \\
\text { stationärer } \\
\text { Aufenthalt }\end{array}$} & ja & 18 & $38,7 \pm 15,8$ & \multirow[b]{2}{*}{$0,0396 *$} & $59,5 \pm 24,9$ & \multirow[b]{2}{*}{$0,023^{*}$} \\
\hline & & nein & 2 & $28,0 \pm 3,4$ & & $34,8 \pm 7,1$ & \\
\hline
\end{tabular}




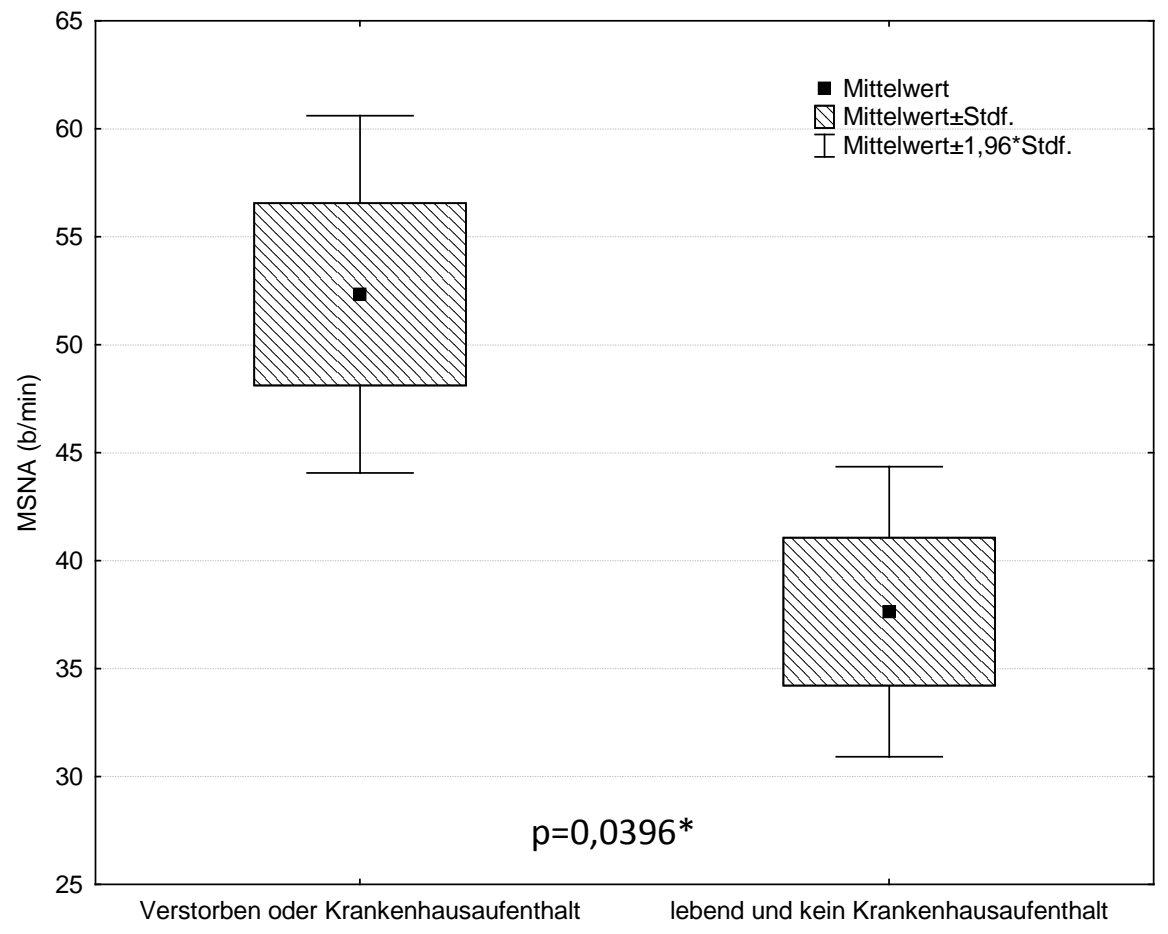

Abbildung 2: Unterschied in der sympathischen Aktivierung (MSNA in b/min) zwischen Herzinsuffizienz-Patienten, die verstorben sind oder mindestens einen Krankenhausaufenthalt hatten und Herzinsuffizienz-Patienten, die weder verstorben sind, noch einen Krankenhausaufenthalt hatten $(38,7 \pm 15,8$ vs. $28,0 \pm 3,4 ; p=0,0396)$

\subsubsection{COPD-Patienten}

\subsubsection{Vergleich der COPD-Patienten mit der Kontrollgruppe}

Zum Zeitpunkt der Befragung waren 6 von 20 COPD-Patienten und einer von 23 Kontrollpersonen verstorben. Bei 4 von 6 verstorbenen COPD-Patienten konnte ein akzidentieller Tod ausgeschlossen werden. Unter den COPD-Patienten gab es signifikant mehr Todesfälle (6 zu 1; p=0,03), mehr Probanden mit Krankenhausaufenthalten (6 zu 1; p=0,018) und ein erhöhtes Mortalitätsrisiko mit einer kürzeren Überlebenszeit ( $\mathrm{p}=0,00079$; siehe Abb. 1).

\subsubsection{Vergleich der COPD-Patienten hinsichtlich klinischer Endpunkte}

Der Grad der sympathischen Aktivierung unterschied sich nicht signifikant zwischen den verstorben und lebenden COPD-Patienten, gemessen als $b / \min (p=0,18)$ und als b/100hbs ( $p=0,13$; siehe Tab. 6). Die bereits verstorbenen Patienten hatten eine signifikant längere Follow-up-Zeit als die Überlebenden (137,0 \pm 3,0 vs. 60,6 \pm 1,9 Monate; $\mathrm{p}=0,00$ ). Die mittlere Überlebenszeit und das Mortalitätsrisiko zwischen den COPD-Patienten, deren sympathische Aktivierung über beziehungsweise unter dem 
Median liegt, unterscheidet sich im Kaplan-Meier-Schätzer ebenfalls nicht signifikant voneinander (b/min $\mathrm{p}=0,47)$.

Bei den befragten COPD-Patienten hatten 6 Patienten seit der mikroneurographischen Untersuchung mindestens einen stationären Aufenthalt aufgrund einer Exazerbation der COPD, 8 Patienten mussten nicht stationär behandelt werden. Die Höhe der sympathischen Aktivierung in b/min zwischen diesen beiden Gruppen war mit einem p-Wert von 0,061 zwar tendenziell, allerdings nicht signifikant erhöht (siehe Tab. 6).

Die Anzahl an Krankenhausaufenthalten reichten von einem bis zu vier (Median=2) und korrelierten nicht mit dem Ausmaß der sympathischen Aktivierung in $\mathrm{b} / \mathrm{min}(\mathrm{p}=0,3)$ oder $\mathrm{b} / 100 \mathrm{hbs}(\mathrm{p}=0,065)$.

Die Patienten, die entweder einen Krankenhausaufenthalt hatten oder verstorben sind ( $n=12)$, haben gegenüber den Patienten, die keines der beiden Ereignisse hatten $(n=8)$, eine signifikant erhöhte sympathische Aktivierung in $b / \min (p=0,022$; siehe Tab.6, Abb. 3), eine signifikant erniedrigte Lungenfunktion, als $\mathrm{FEV}_{1}$-Ist/FEV ${ }_{1}$-Soll $(\mathrm{p}=0,035)$ und als $\mathrm{pO} 2(\mathrm{p}=0,025)$ in der arteriellen Blutgasanalyse, sowie eine signifikant erhöhte Herzfrequenz ( $p=0,017$; siehe Tab 7). Die Patienten in der Gruppe mit kombiniertem Endpunkt hatten signifikant längeres Follow-Up (98,5 \pm 40,3 vs. 61,1 \pm 2,1 Monate; $\mathrm{p}=0,01)$. 


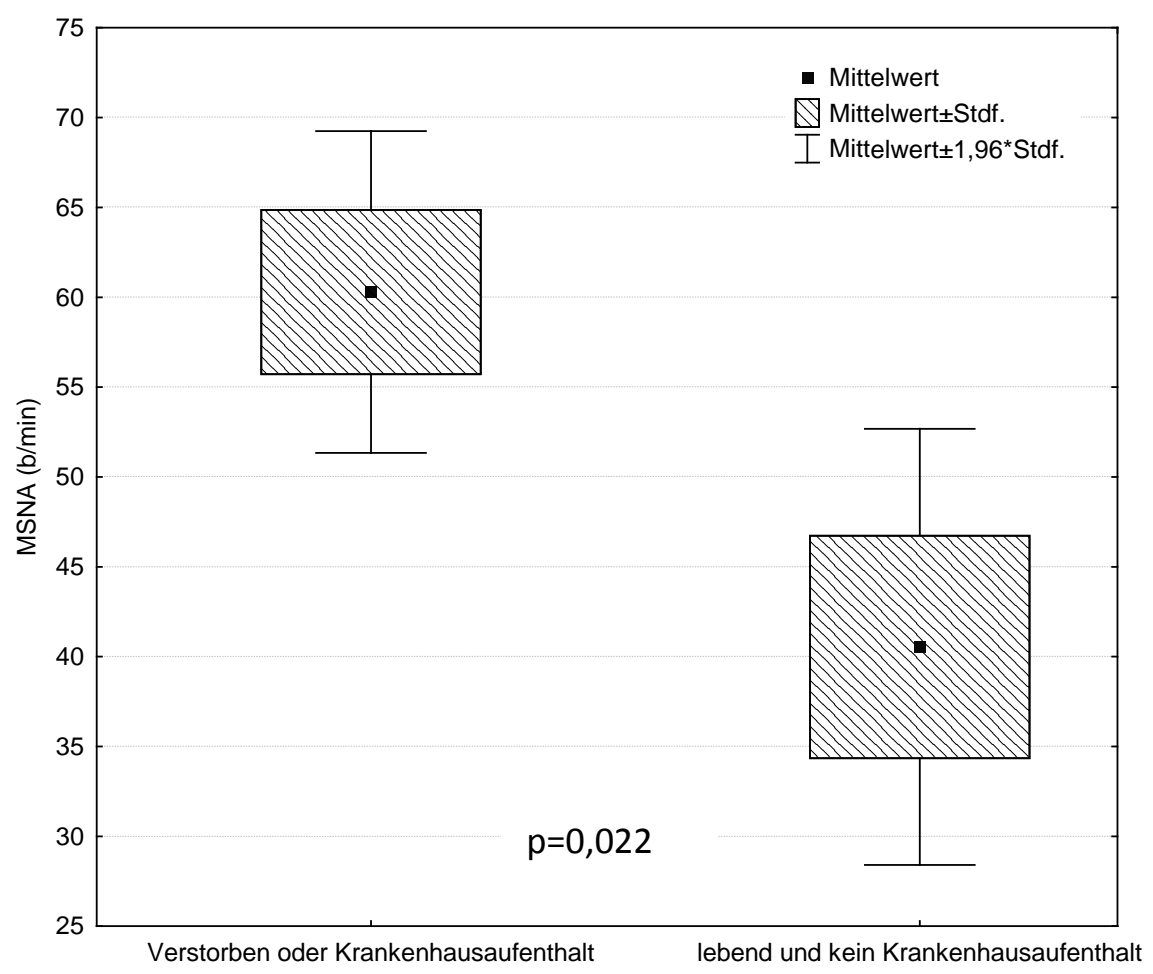

Abbildung 3: Unterschied in der sympathischen Aktivierung (MSNA in b/min) zwischen COPD-Patienten, die verstorben sind oder mindestens einen Krankenhausaufenthalt hatten und COPD-Patienten, die weder verstorben sind, noch einen Krankenhausaufenthalt hatten (60,3 $\pm 15,8$ vs. 40,5 $\pm 17,5 ; p=0,022)$

Tabelle 6: Vergleich der sympathischen Aktivierung der COPD-Patienten aufgeteilt nach klinischen Endpunkten

\begin{tabular}{|c|c|c|c|c|c|c|c|}
\hline & & & Anzahl (n) & b/min & p-Wert & b/100hbs & p-Wert \\
\hline \multirow{6}{*}{$\begin{array}{l}\text { Klinischer } \\
\text { Endpunkt }\end{array}$} & \multirow{2}{*}{ Tod } & ja & 6 & $60,8 \pm 16,4$ & \multirow{2}{*}{0,18} & $69,6 \pm 10,0$ & \multirow{2}{*}{0,13} \\
\hline & & nein & 14 & $48,7 \pm 19,2$ & & $59,8 \pm 17,7$ & \\
\hline & \multirow{2}{*}{$\begin{array}{l}\text { Stationärer } \\
\text { Aufenthalt }\end{array}$} & ja & 6 & $59,7 \pm 16,7$ & \multirow{2}{*}{0,061} & $68,3 \pm 12,8$ & \multirow{2}{*}{0,10} \\
\hline & & nein & 8 & $40,5 \pm 17,5$ & & $53,4 \pm 18,8$ & \\
\hline & \multirow{2}{*}{$\begin{array}{l}\text { Tod oder } \\
\text { stationärer } \\
\text { Aufenthalt }\end{array}$} & ja & 12 & $60,3 \pm 15,8$ & \multirow[b]{2}{*}{$0,022 *$} & $69,0 \pm 11,0$ & \multirow[b]{2}{*}{0,0598} \\
\hline & & nein & 8 & $40,5 \pm 17,5$ & & $53,4 \pm 18,8$ & \\
\hline
\end{tabular}


Tabelle 7: Vergleich von COPD-Patienten, die entweder verstorben sind oder stationär behandelt werden mussten, mit COPD-Patienten, die weder verstorben sind noch stationär behandelt werden mussten

\begin{tabular}{|c|c|c|c|}
\hline & $\begin{array}{c}\text { Tod oder stationärer } \\
\text { Aufenthalt }\end{array}$ & $\begin{array}{c}\text { nicht verstorben, kein } \\
\text { stationärer Aufenthalt }\end{array}$ & p-Wert (2-seitig) \\
\hline Anzahl (n) & 12 & 8 & 0,25 \\
\hline Alter (Jahren) & $63,4 \pm 8,9$ & $59,8 \pm 4,8$ & 0,73 \\
\hline Körpergröße (cm) & $171,4 \pm 0,1$ & $170,0 \pm 0,1$ & 0,14 \\
\hline Gewicht (kg) & $74,9 \pm 16,7$ & $83,8 \pm 8,8$ & 0,087 \\
\hline Body-Mass-Index & $25,4 \pm 5,0$ & $29,1 \pm 4,1$ & $0,035^{*}$ \\
\hline $\begin{array}{c}\text { FEV } \text {-Ist/FEV } \text {-Soll } \\
\text { (in \% der Norm) }\end{array}$ & $39 \pm 10$ & $54 \pm 10$ & $0,025^{*}$ \\
\hline pO $_{\mathbf{2}}$ (art. in mmHg) & $69,1 \pm 10,9$ & $78,0 \pm 5,1$ & 0,43 \\
\hline pCO $_{\mathbf{2}}$ (art. in mmHg) & $42,5 \pm 4,3$ & $41,15 \pm 2,9$ & $0,017^{*}$ \\
\hline $\begin{array}{c}\text { Herzfrequenz } \\
\text { (Schläge/min) }\end{array}$ & $90,5 \pm 18,9$ & $73,6 \pm 9,5$ & $0,022^{*}$ \\
\hline MSNA (b/min) & $60,3 \pm 15,8$ & $40,5 \pm 17,5$ & 0,0598 \\
\hline MSNA (b/100hbs) & $69,0 \pm 11,0$ & $53,4 \pm 18,8$ & \\
\hline
\end{tabular}




\section{DISKUSSION}

\subsection{Herzinsuffizienz-Patienten}

Die Herzinsuffizienz-Patienten wiesen im Vergleich zu den Kontrollpersonen eine erhöhte sympathische Aktivierung sowohl in b/min als auch b/100hbs auf (siehe Tab.3). Die erhöhte sympathische Aktivierung ließ sich feststellen, obwohl alle Patienten zur Zeit der mikroneurographischen Untersuchung mindestens eine sympathoinhibitorische Dauermedikation, wie ACE-Hemmer und/oder $\beta$-Blocker, erhielten. Zudem wiesen die Patienten gegenüber der Kontrollgruppe eine wie erwartet erhöhte Morbidität und Mortalität mit einer kürzeren Überlebenszeit auf (siehe Abb. 1). Im Vergleich zu den gesunden Kontrollpersonen lassen die Ergebnisse der Befragung auf einen typischen Krankheitsverlauf der Herzinsuffizienz mit einer signifikant erhöhten Wahrscheinlichkeit eines Krankenhausaufenthalts und einer erhöhten Sterblichkeit mit kürzeren Überlebenszeiten (siehe Abb. 1) schließen.

Um die Auswirkungen des Grades der sympathischen Aktivierung bei den Patienten zu beurteilen, wurden diese bezüglich der klinischen Endpunkte in Subgruppen unterteilt.

Bei verstorbenen Herzinsuffizienz-Patienten war der Grad der sympathischen Aktivierung gegenüber den Lebenden, gemessen als $b / \mathrm{min}$, entgegen der Erwartung signifikant erniedrigt $(p=0,038$; siehe Tab. 5). Es konnte kein Unterschied im Mortalitätsrisiko zwischen den Patienten festgestellt werden, deren sympathische Aktivität über beziehungsweise unter dem Median lag.

Die Patienten, die mindestens einmal stationär behandelt werden mussten, hatten gegenüber den Patienten, die nicht stationär behandelt werden mussten, eine signifikant erhöhte sympathische Aktivität in $\mathrm{b} / \mathrm{min}(\mathrm{p}=0,0062)$ und $\mathrm{b} / 100 \mathrm{hbs}$ ( $\mathrm{p}=0,007$; siehe Tab. 5).

Da bei der alleinigen Betrachtung des Krankenhausaufenthalts Patienten vernachlässigt werden, bei denen das für den Krankheitsverlauf bedeutendere Ereignis des Todes eingetreten ist, wurden die Patienten in einer Gruppe zusammengefasst, die als zusammengesetzten Endpunkt entweder bereits verstorben waren oder mindestens einen Krankenhausaufenthalt hatten und mit Patienten verglichen, die weder verstorben sind, noch einen Krankenhausaufenthalt hatten.

Bei den Patienten, die verstorben sind oder einen Krankenhausaufenthalt hatten, war die sympathische Aktivität in $b / \min (p=0,0396)$ und $b / 100 h b s(p=0,023)$ gegenüber Patienten ohne ein entsprechendes Ereignis signifikant erhöht (siehe Tab. 5, Abb. 2). Allerdings muss hier einschränkend gesagt werden, dass aufgrund der geringen Anzahl von Patienten, die jeweils kein Ereignis hatten $(n=2)$, auch bei statistischer Signifikanz die Aussagekraft begrenzt ist. 
Im Gegensatz zur COPD gibt es bei der Herzinsuffizienz bereits Studien, die die Auswirkungen der sympathischen Aktivierung auf die Mortalität untersucht haben.

In einer kontrollierten Studie von Barretto et al. wurde bei 122 Herzinsuffizienz-Patienten die sympathische Aktivierung mikroneurographisch gemessen und in einem Follow-up von einem Jahr in Bezug zur Sterblichkeit gesetzt. Die Gruppe der verstorbenen Patienten wies gegenüber den lebenden Patienten eine signifikant erhöhte sympathische Aktivierung sowohl in b/min als auch b/100hbs auf. Darüber hinaus zeigte sich in der multivariaten Analyse, dass die mikroneurographisch gemessene sympathische Aktivierung ein unabhängiger Prädiktor der Mortalität und dabei selbst der linksventrikulären Ejektionsfraktion überlegen war (Binggeli et al. 2002; Barretto et al. 2009 (Binggeli et al. 2002; Cohn et al. 1984; Grassi et al. 2009)).

In einer weiteren kontrollierten Studie von Munhoz et al. wurde die Assoziation des Grades der mikroneurographisch gemessenen sympathischen Aktivierung in Hinsicht auf Schwere der Herzinsuffizienz (NYHA) und Mortalität an 52 Patienten im 18-monatigen Follow-Up untersucht. Die verstorbenen Patienten wiesen bei gleicher funktionaler Schwere (NYHA) eine signifikant erhöhte sympathische Aktivierung gegenüber den lebenden Patienten auf. Darüber hinaus korrelierte das Ausmaß der sympathischen Aktivierung signifikant mit dem NYHA-Grad (Munhoz et al. 2009).

Die allgemeinen Charakteristika der Patienten beider Studien, die Medikation, sowie die mikroneurographischen Messungen waren zum Zeitpunkt der Untersuchung vergleichbar mit denen der hier durchgeführten Studie. Allerdings wiesen die Patienten in beiden Studien mit einer mittleren sympathischen Aktivität von 50,6 b/min bzw. 53,1 b/min eine im Schnitt deutlich höhere sympathische Aktivierung auf, als die Herzinsuffizienzpatienten unserer Studie mit 37,7 b/min (siehe Tab.4; (Barretto et al. 2009; Munhoz et al. 2009).

Beide beschriebenen Studien sind durch das kontrollierte Studiendesign und die höheren Fallzahlen unserer Studie in der Evidenz hinsichtlich der Mortalität überlegen. Beachtet werden muss neben den höheren Fallzahlen allerdings auch die im Mittelwert deutlich erhöhte sympathische Aktivierung gegenüber den Patienten unserer Studie. Aus den allgemeinen Charakteristika und der Medikation zum Zeitpunkt der Untersuchung lässt sich dieser Sachverhalt nicht erklären. Die stärkere sympathische Aktivierung könnte daher neben den höheren Fallzahlen zur Signifikanz der Ergebnisse beigetragen haben.

Die beobachtete erniedrigte sympathische Aktivierung bei verstorbenen Herzinsuffizienz-Patienten gegenüber den lebenden Patienten lässt sich in Anbetracht der oben erwähnten Studien sowie der pathophysiologischen Grundlagen nicht nachvollziehen (siehe Tab. 5). 
Ein wichtiger Aspekt, der in diesem Zusammenhang eine Rolle spielen könnte, sind individuelle Konditionen der Patienten hinsichtlich der Ausprägung der sympathischen Aktivierung, welche insbesondere bei niedrigen Fallzahlen Auswirkungen auf die Ergebnisse haben könnten.

Wie oben bereits beschrieben ist die sympathische Aktivierung kein statischer Wert, der lediglich von der funktionellen Schwere der Herzinsuffizienz abhängt, auch wenn diese direkt mit den klinischen Endpunkten assoziiert ist (Munhoz et al. 2009; Patel und Zheng 2012).

Durch den Ausschluss von Sympathikus-fördernden Komorbiditäten und Medikationen konnten die meisten Störfaktoren bereits ausgeschlossen werden. Allerdings war es durch das zugrundeliegende Studiendesign nicht möglich die konditionellen Voraussetzungen der Patienten, welche signifikanten Einfluss auf den Grad der sympathischen Aktivierung haben, zu bewerten (Li YL et al. 2008; Liu et al. 2000; Roveda et al. 2003).

Unsere Studie erfasst, gegenüber den vorgestellten Studien, die Anzahl der Krankenhausaufenthalte als validen Parameter zur Abschätzung der Morbidität und den zusammengesetzten Endpunkt aus Krankenhausaufenthalt oder Tod. Wenn auch durch die Fallzahl eingeschränkt aussagekräftig, zeigte sich hier die Assoziation einer erhöhten sympathischen Aktivierung mit einer gesteigerten Morbidität alleine und als zusammengesetztem Endpunkt gesteigerter Morbidität und Mortalität (siehe Tab. 5).

Diese Beobachtung lässt sich sowohl durch die oben beschriebenen pathophysiologischen Mechanismen einer erhöhten sympathischen Aktivität logisch nachvollziehen als auch durch den positiven Effekt einer Hemmung der sympathischen Aktivität durch die gängigen Therapien der Herzinsuffizienz und steht im Einklang mit dem momentanen Wissenstand (De Matos et al. 2004; Krum und Teerlink 2011).

Zusammenfassend zeigt die Nachbefragung der Herzinsuffizienz-Patienten eine Assoziation der erhöhten sympathischen Aktivierung mit verstärkter Morbidität, die auch mit der pathophysiologischen Sicht und den Erkenntnissen der aktuellen Therapie übereinstimmt. Die Assoziation mit einer erhöhten Mortalität als einzelner Endpunkt, wie sie schon in umfangreicheren Studien gezeigt wurde, konnte durch die Ergebnisse unserer Studie nicht oder nur eingeschränkt bestätigt werden. 


\subsection{COPD-Patienten}

Die in der Studie befragten COPD-Patienten zeigten gegenüber der Kontrollgruppe die krankheitstypischen Charakteristika. So gab es bei den Lungenfunktionsergebnissen signifikante Anzeichen einer Obstruktion und eine signifikant erhöhte sympathische Aktivierung (siehe Tab. 3). Die Nachbefragung bestätigte gegenüber dem gesunden Kontrollkollektiv einen typischen, mit der Literatur übereinstimmenden Krankheitsverlauf mit einer signifikant erhöhten Wahrscheinlichkeit eines Krankenhausaufenthalts $(\mathrm{p}=0,018)$ und einer erhöhten Sterblichkeit mit kürzeren Überlebenszeiten ( $\mathrm{p}=0,00079$; siehe Abb. 1; Rabe et al. 2007).

Die COPD-Patienten wurden ebenfalls hinsichtlich der klinischen Endpunkte miteinander verglichen.

Die Patienten, die bereits verstorben waren, hatten gegenüber den noch lebenden Patienten eine zwar im Mittelwert erhöhte sympathische Aktivierung (b/min 60,8 \pm 16,4 vs. 48,7 \pm 19,2), allerdings ohne statistische Signifikanz ( $\mathrm{p}=0,18$; siehe Tab. 6). Das Mortalitätsrisiko der Patienten mit einer sympathischen Aktivierung über beziehungsweise unter dem Median unterschied sich nicht signifikant voneinander.

Insbesondere bei der Betrachtung der Mortalität ist die unterschiedlich lange Follow-Up-Zeit der COPD-Patienten zu beachten. Die verstorbenen COPD-Patienten hatten ein signifikant längeres Follow-Up. Auch wenn die verstorbenen Patienten tendenziell eine im Mittelwert erhöhte sympathische Aktivität aufwiesen, ist die Aussagekraft bezüglich dieses einzelnen klinischen Endpunkts nur eingeschränkt zu interpretieren. Daher lässt sich bei unserer Studie kein statistisch signifikanter Zusammenhang von gemessener sympathischer Aktivierung und Mortalität als einzelner Endpunkt feststellen.

Die Patienten, die seit der mikroneurographischen Messung einen Krankenhausaufenthalt aufgrund ihrer COPD hatten, zeigten gegenüber den Patienten, die keinen Krankenhausaufenthalt hatten, eine im Mittelwert erhöhte (b/min 59,7 vs. 40,5), aber nicht statistisch signifikante sympathische Aktivierung mit einem p-Wert von 0,061 (siehe Tab. 6). Der Grad der sympathischen Aktivierung korrelierte nicht mit der Anzahl der Krankenhausaufenthalte in $b / \min (p=0,3)$ und b/100hbs ( $p=0,065)$.

Bei der Betrachtung der einzelnen klinischen Endpunkte ließ sich durch die höheren Mittelwerte bei verstorbenen Patienten oder Patienten mit Krankenhausaufenthalt ein tendenziell erhöhter Grad der sympathischen Aktivierung beobachten (siehe Tab. 6). Jedoch konnten diese Ergebnisse, eventuell durch die zugrundeliegende und durch das Studiendesign begrenzte Stichprobenanzahl, nicht statistisch signifikant abgesichert werden.

Da das Studiendesign und der innovative Ansatz nur eine geringe Fallzahl vorgaben, wurde zur Erhöhung der statistischen Präzision ein zusammengesetzter klinischer Endpunkt verwendet 
(Krankenhausaufenthalt oder Tod im Follow-Up). Die beiden einzelnen Endpunkte haben jeweils eine bedeutende klinische Relevanz für den Patienten und stehen darüber hinaus in einer klaren pathophysiologischen Beziehung zueinander mit einer deutlichen Erhöhung der Mortalität durch häufigere Exazerbationen (Soler-Cataluna et al. 2005; Seemungal et al. 2009). Zudem besteht eine bereits beschriebene biologische Plausibilität möglicher pathophysiologischer Auswirkungen einer erhöhten sympathischen Aktivität auf beide einzelne Endpunkte.

Patienten, die entweder einen Krankenhausaufenthalt hatten, oder verstorben sind, zeigten eine signifikant deutlich erhöhte sympathische Aktivierung in b/min (60,3 vs. 40,5; $\mathrm{p}=0,022$; siehe Abb. 3), des Weiteren ein niedrigeres $\mathrm{FEV}_{1}$-Ist/ $\mathrm{FEV}_{1}$-Soll (0,39 vs. 0,54; $\left.\mathrm{p}=0,035\right)$, niedrigeren $\mathrm{pO}_{2}$ (69,12 vs. 78,04; $\mathrm{p}=0,025)$ und eine erhöhte Herzfrequenz (90,48 vs. 73,62; $\mathrm{p}=0,017$; siehe Tab 7.).

Die erhöhten Herzfrequenzen bei morbideren Patienten können als Ausdruck der erhöhten sympathischen Aktivierung gesehen werden und bestätigen als physiologischer Marker die Effekte der verstärkten sympathischen Aktivierung. Auch bei dem zusammengesetzten klinischen Endpunkt wirkt die unterschiedliche Follow-Up-Zeit der COPD-Patienten als möglicher Confounder, allerdings erklärt dies nicht eine signifikant erhöhte sympathische Aktivierung bei verstorbenen oder hospitalisierten Patienten.

Von der pathophysiologischen Seite gibt es einige Hinweise, die einen direkten und indirekten Effekt der sympathischen Aktivierung auf die pulmonale Leistungsfähigkeit vermuten lassen.

Wie bereits im Detail besprochen gibt es Anhaltspunkte dafür, dass die sympathische Aktivierung zu Kachexie und Muskelatrophie führende Prozesse fördert und somit das pathologische Remodeling des Atemapparats vorantreibt (Anker und Coats 1999; Wagner 2008; Wosten-van Asperen et al. 2008).

Zusätzlich könnte eine chronisch erhöhte sympathische Aktivierung zur Desensibilisierung der pulmonalen Betarezeptoren beitragen und so die Obstruktion fördern, sowie die antiobstruktive Therapie erschweren (Seals und Dinenno 2004; Weiss und Shore 2004).

Es ist demnach möglich, dass die signifikant erniedrigten $\mathrm{pO}_{2}$ - und $\mathrm{FEV}_{1}$-Werte bei Patienten, die im Laufe des Follow-Up-Zeitraums verstorben sind oder einen Krankenhausaufenthalt hatten, unter anderem in Verbindung stehen mit der erhöhten sympathischen Aktivierung. Allerdings korrelierte die

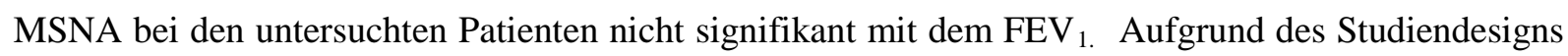
und der Fallzahlen lassen sich diesbezüglich daher nur begrenzt Aussagen treffen. Da das $\mathrm{FEV}_{1}$ direkt mit dem Schweregrad und der Prognose der COPD korreliert, müsste dieser Zusammenhang in zukünftigen Untersuchungen genauer evaluiert werden.

In der Literatur gab es bisher keine Untersuchungen, inwiefern eine mikroneurographisch gemessene, erhöhte sympathische Aktivierung bei COPD-Patienten mit erhöhter Morbidität und Mortalität assoziiert ist. Aus den dargestellten Vergleichen mit der Herzinsuffizienz, bei der eine erhöhte sympathische Aktivierung zu einer nachweislich schlechteren Prognose führt, lässt sich auch ein 
ähnlicher Effekt bei der COPD annehmen (Andreas et al. 2005; Barretto et al. 2009; Munhoz et al. 2009).

In mehreren Studien konnten Hinweise dafür gefunden werden, dass sich Therapien mit ACEHemmern, $\mathrm{AT}_{\mathrm{II}^{-}}$, oder Betablockern, die die Sympathikusaktivität erwiesenermaßen signifikant hemmen, auch bei COPD-Patienten sowohl auf die Morbidität, als auch auf die Mortalität günstig auswirken.

Mancini et al. konnten in einer retrospektiven Studie bereits zeigen, dass die Therapie mit ACEHemmern oder $\mathrm{AT}_{\mathrm{II}}$-Blockern, unabhängig vom Grad des kardiovaskulären Risikos der Patienten, sowohl die Morbidität, als auch die Mortalität bei COPD senkte (Mancini 2005; Mancini et al. 2006). In zwei weiteren umfassenden retrospektiven Studien von Rutten et al. und Short et al. wurde kürzlich die Auswirkungen einer Betablocker-Therapie auf Morbidität und Mortalität von COPD-Patienten untersucht. Bisher galten Betablocker aufgrund potentiell bronchokonstriktorischer Eigenschaften und vermeintlicher kompetitiver Wirkung auf inhalative Betamimetika bei COPD-Patienten als problematisch (Egred et al. 2005; Ling et al. 2008). Es zeigte sich jedoch, dass insbesondere kardioselektive Betablocker keinen andauernden negativen Effekt auf das $\mathrm{FEV}_{1}$, die Ansprechbarkeit auf inhalative Betamimetika und respiratorische Symptome hatten und selbst von Patienten mit asthmatischer COPD-Komponente gut toleriert wurden (Dransfield et al. 2008; Lin et al. 2008; Navas und Taylor 2010; Salpeter et al. 2002a; Salpeter et al. 2002b).

In beiden Studien korrelierte die Betablocker-Therapie mit einer signifikanten Abnahme der Mortalität und Morbidität, beziehungsweise einem Rückgang der Häufigkeit von Krankenhauseinweisungen und Exazerbationen, auch hier unabhängig vom kardiovaskulären Risikoprofil. In der Studie von Rutten et al. hatte die Subgruppe von Patienten, bei denen bei der Diagnose COPD die Betablocker-Therapie abgesetzt wurde, sogar die höchste Mortalität (Au et al. 2004; Rutten et al. 2010; Short et al. 2011).

Die Evaluation der pathophysiologischen Auswirkungen der sympathischen Aktivierung auf die Prognose der COPD steht bisher noch am Anfang. Es gibt aber bereits Hinweise, die für eine Ergänzung der bisherigen, lediglich symptomatischen COPD-Therapien sprechen, hin zu einer kausaleren, insbesondere auch die sympathischen Komponenten betreffenden Therapie.

Die in dieser Studie gewonnenen Erkenntnisse stützen zusammen mit den beschriebenen Forschungsergebnissen anderer Arbeitsgruppen die These eines die Prognose verschlechternden Effekts der erhöhten sympathischen Aktivierung bei chronisch obstruktiver Lungenerkrankung.

Um eine genauere Charakterisierung des Grades der sympathischen Aktivierung bei COPD-Patienten und die Auswirkungen möglicher sympathoinhibitorischer Therapien zu untersuchen, müssten zukünftig kontrollierte Längsschnittstudien mit größeren Fallzahlen und möglichst repetitiver Messung der sympathischen Aktivierung durchgeführt werden. 


\subsection{Einschränkungen}

Durch das Studiendesign war die Stichprobenzahl bereits vorgegeben, sodass durch den geringen Umfang eventuelle Zusammenhänge nicht statistisch signifikant abgesichert werde konnten ( $\alpha$-Fehler) oder die Möglichkeit bestand eine falsche statistische Signifikanz aufgrund einer zu geringen Stichprobenzahl zu erhalten ( $\beta$-Fehler). Weiterhin war eine kontrollierte Betrachtung des Verlaufs, insbesondere wechselnder Medikationen mit eventuell wechselnden Graden der sympathischen Aktivität, nicht evaluierbar. Auch konnte die Todesursachen nur der meisten, aber nicht aller Verstorbenen auf die jeweilige Erkrankung eingegrenzt werden.

\section{ZUSAMMENFASSUNG}

Die chronisch obstruktive Lungenerkrankung (COPD) stellt sowohl aus medizinischer als auch sozioökonomischer Sicht eine wachsende globale Herausforderung dar (Gershon et al. 2011; Rabe et al. 2007). Bislang existieren allerdings lediglich symptomatische Therapien, die der Bedeutung und Schwere der Erkrankung bisher nicht gerecht werden konnten.

Mit dem Nachweis einer erhöhten sympathischen Aktivierung bei der COPD konnte in der Vergangenheit ein weiteres Mal der systemische Charakter der Erkrankung unterstrichen und die komplexen pathophysiologischen Interaktionen um einen zusätzlichen Aspekt ergänzt werden (Heindl et al. 2001; Raupach et al. 2008).

Von Erkrankungen, wie der Herzinsuffizienz, die ebenfalls mit einer erhöhten sympathischen Aktivierung einhergehen und pathophysiologischen Beobachtungen, lässt sich ein die Prognose verschlechternder Effekt der erhöhten sympathischen Aktivierung annehmen (Andreas et al. 2005; Barretto et al. 2009; Munhoz et al. 2009). Dieser beruht unter anderem auf einer verstärkten Kachexie, gesteigertem kardiovaskulärem Risiko und einer Forcierung des pathologischen Remodeling der Lunge und der Atemmuskulatur mit konsekutiver Verschlechterung der respiratorischen Situation.

In dieser Studie wurde in einem Follow-Up 20 Herzinsuffizienz-Patienten, 20 COPD-Patienten und 23 Kontrollpersonen, die initial mikroneurographisch untersucht wurden, telefonisch nach Krankenhausaufenthalten aufgrund ihrer Grunderkrankung befragt, beziehungsweise der Todeszeitpunkt ermittelt. Nach erfolgter statistischer Auswertung wurden die entsprechenden Kollektive nach klinischen Endpunkten in Subgruppen unterteilt und der Grad der initial gemessenen sympathischen Aktivierung verglichen.

Sowohl Herzinsuffizienz- als auch COPD-Patienten, die aufgrund ihrer Erkrankung einen Krankenhausaufenthalt hatten oder verstarben, wiesen gegenüber Patienten, die keinen 
Krankenhausaufenthalt hatten, eine signifikant erhöhte sympathische Aktivierung auf (siehe Abb. 2 und 3). Durch diese Studie konnte bei COPD-Patienten erstmalig eine signifikante Assoziation einer erhöhten sympathischen Aktivierung mit einer gesteigerten Morbidität und Mortalität nachgewiesen werden.

Zu diesem Zeitpunkt steht die Forschung bezüglich der Auswirkungen der erhöhten sympathischen Aktivierung noch am Anfang. Um die gewonnenen Ergebnisse abzusichern und den Grad und die Auswirkungen der erhöhten sympathischen Aktivierung genauer zu evaluieren, müssten zukünftig kontrollierte Studien mit größeren Fallzahlen durchgeführt werden.

Nach ausführlicheren Untersuchungen bietet dieses noch junge Forschungsfeld perspektivisch eventuell die Möglichkeit, die bisherigen COPD-Therapien um eine kausalere, die Prognose potentiell verbessernde Therapie zu ergänzen. Erste umfangreichere, wenn auch nur retrospektive Studien konnten bereits einen positiven Effekt einer sympathoinhibitorischen Medikation mit ACE-Hemmern, $\mathrm{AT}_{\mathrm{II}}{ }^{-}$oder $\beta$-Blockern auf Morbidität und Mortalität bei COPD zeigen (Andreas et al. 2006; Mancini et al. 2006; Rutten et al. 2010; Short et al. 2011). 


\section{LITERATURVERZEICHNIS}

Adams KF, Jr. (2004): Pathophysiologic role of the renin-angiotensin-aldosterone and sympathetic nervous systems in heart failure. Am J Health Syst Pharm 61 Suppl 2, S4-13

Adamson PB und EM Gilbert (2006): Reducing the risk of sudden death in heart failure with betablockers. J Card Fail 12, 734-46

Agusti AG, A Noguera, J Sauleda, E Sala, J Pons und X Busquets (2003): Systemic effects of chronic obstructive pulmonary disease. Eur Respir J 21, 347-60

Agusti A, M Morla, J Sauleda, C Saus und X Busquets (2004): NF-kappaB activation and iNOS upregulation in skeletal muscle of patients with COPD and low body weight. Thorax $\underline{59}$, 4837

Andreas S, H Reiter, L Luthje, A Delekat, RW Grunewald, G Hasenfuss und VK Somers (2004): Differential effects of theophylline on sympathetic excitation, hemodynamics, and breathing in congestive heart failure. Circulation 110, 2157-62

Andreas S, SD Anker, PD Scanlon und VK Somers (2005): Neurohumoral activation as a link to systemic manifestations of chronic lung disease. Chest $\underline{128}, 3618-24$

Andreas S, C Herrmann-Lingen, T Raupach, L Luthje, JA Fabricius, N Hruska, W Korber, B Buchner, CP Criee, G Hasenfuss und P Calverley (2006): Angiotensin II blockers in obstructive pulmonary disease: a randomised controlled trial. Eur Respir J 27, 972-9

Andreas S, T Hering, S Muhlig, D Nowak, T Raupach und H Worth (2009): Smoking cessation in chronic obstructive pulmonary disease: an effective medical intervention. Dtsch Arztebl Int 106, 276-82

Anker SD und AJ Coats (1999): Cardiac cachexia: a syndrome with impaired survival and immune and neuroendocrine activation. Chest $\underline{115}, 836-47$

Anker SD und R Sharma (2002): The syndrome of cardiac cachexia Int J Cardiol 85, 51-66

Au DH, CL Bryson, VS Fan, EM Udris, JR Curtis, MB McDonell und SD Fihn (2004): Beta-blockers as single-agent therapy for hypertension and the risk of mortality among patients with chronic obstructive pulmonary disease. Am J Med 117, 925-31

Barbera JA, VI Peinado und S Santos (2003): Pulmonary hypertension in chronic obstructive pulmonary disease. Eur Respir J 21, 892-905

Barnes PJ (2008a): The cytokine network in asthma and chronic obstructive pulmonary disease. J Clin Invest $\underline{118}, 3546-56$

Barnes PJ (2008b): Immunology of asthma and chronic obstructive pulmonary disease. Nat Rev Immunol 8, 183-92

Barnes PJ und BR Celli (2009): Systemic manifestations and comorbidities of COPD. Eur Respir J $\underline{33}$, 1165-85 
Barretto AC, AC Santos, R Munhoz, MU Rondon, FG Franco, IC Trombetta, F Roveda, LN de Matos, AM Braga, HR Middlekauff und CE Negrao (2009): Increased muscle sympathetic nerve activity predicts mortality in heart failure patients. Int J Cardiol 135, 302-7

Bartels MN, JM Gonzalez, W Kim und RE De Meersman (2000): Oxygen supplementation and cardiac-autonomic modulation in COPD. Chest $\underline{118}, 691-6$

Bedard ME, K Marquis, P Poirier und S Provencher (2010): Reduced heart rate variability in patients with chronic obstructive pulmonary disease independent of anticholinergic or beta-agonist medications. COPD $\underline{7}, 391-7$

Beeh KM, O Kornmann, R Buhl, SV Culpitt, MA Giembycz und PJ Barnes (2003): Neutrophil chemotactic activity of sputum from patients with COPD: role of interleukin 8 and leukotriene B4. Chest 123, 1240-7

Bernardi L, A Gabutti, C Porta und L Spicuzza (2001): Slow breathing reduces chemoreflex response to hypoxia and hypercapnia, and increases baroreflex sensitivity. J Hypertens $\underline{19}$, 2221-9

Bernardi L, C Porta, L Spicuzza, J Bellwon, G Spadacini, AW Frey, LY Yeung, JE Sanderson, R Pedretti und R Tramarin (2002): Slow breathing increases arterial baroreflex sensitivity in patients with chronic heart failure. Circulation $\underline{105}$, 143-5

Binggeli C, R Corti, I Sudano, TF Luscher und G Noll (2002): Effects of chronic calcium channel blockade on sympathetic nerve activity in hypertension. Hypertension 39, 892-6

Blanc PD, C Iribarren, L Trupin, G Earnest, PP Katz, J Balmes, S Sidney und MD Eisner (2009): Occupational exposures and the risk of COPD: dusty trades revisited. Thorax $\underline{64}, 6-12$

Borst MM, W Beuthien, C Schwencke, P LaRosee, R Marquetant, M Haass, W Kubler und RH Strasser (1999): Desensitization of the pulmonary adenylyl cyclase system: a cause of airway hyperresponsiveness in congestive heart failure?. J Am Coll Cardiol 34, 848-56

Bourdin A, PR Burgel, P Chanez, G Garcia, T Perez und N Roche (2009): Recent advances in COPD: pathophysiology, respiratory physiology and clinical aspects, including comorbidities. Eur Respir Rev 18, 198-212

Boutou AK, I Stanopoulos, GG Pitsiou, T Kontakiotis, G Kyriazis, L Sichletidis und P Argyropoulou (2011): Anemia of chronic disease in chronic obstructive pulmonary disease: a case-control study of cardiopulmonary exercise responses. Respiration $\underline{82}$, 237-45

Bratel T, A Wennlund und K Carlstrom (2000): Impact of hypoxaemia on neuroendocrine function and catecholamine secretion in chronic obstructive pulmonary disease (COPD). Effects of long-term oxygen treatment. Respir Med 94, 1221-8

Bristow MR, R Ginsburg, W Minobe, RS Cubicciotti, WS Sageman, K Lurie, ME Billingham, DC Harrison und EB Stinson (1982): Decreased catecholamine sensitivity and beta-adrenergicreceptor density in failing human hearts. N Engl J Med 307, 205-11

Broekhuizen R, JH Vernooy, AM Schols, MA Dentener und EF Wouters (2005): Leptin as local inflammatory marker in COPD. Respir Med $\underline{99}$, 70-4 
Brouwer J, DJ van Veldhuisen, AJ Man in 't Veld, J Haaksma, WA Dijk, KR Visser, F Boomsma und PH Dunselman (1996): Prognostic value of heart rate variability during long-term follow-up in patients with mild to moderate heart failure. The Dutch Ibopamine Multicenter Trial Study Group. J Am Coll Cardiol 28, 1183-9

Brum PC, AV Bacurau, A Medeiros, JC Ferreira, AS Vanzelli und CE Negrao (2011): Aerobic exercise training in heart failure: impact on sympathetic hyperactivity and cardiac and skeletal muscle function. Braz J Med Biol Res 44, 827-35

Bruno A, S Conus, I Schmid und HU Simon (2005a): Apoptotic pathways are inhibited by leptin receptor activation in neutrophils. J Immunol 174, 8090-6

Bruno A, P Chanez, G Chiappara, L Siena, S Giammanco, M Gjomarkaj, G Bonsignore, J Bousquet und AM Vignola (2005b): Does leptin play a cytokine-like role within the airways of COPD patients?. Eur Respir J 26, 398-405

Buist AS, MA McBurnie, WM Vollmer, S Gillespie, P Burney, DM Mannino, AM Menezes, SD Sullivan, TA Lee, KB Weiss, RL Jensen, GB Marks, A Gulsvik und E NizankowskaMogilnicka (2007): International variation in the prevalence of COPD (the BOLD Study): a population-based prevalence study. Lancet $\underline{370}$, 741-50

Calbet JA (2003): Chronic hypoxia increases blood pressure and noradrenaline spillover in healthy humans. J Physiol 551, 379-86

Caldefie-Chezet F, A Poulin, A Tridon, B Sion und MP Vasson (2001): Leptin: a potential regulator of polymorphonuclear neutrophil bactericidal action?. J Leukoc Biol $\underline{69}$, 414-8

Calikoglu M, G Sahin, A Unlu, C Ozturk, L Tamer, B Ercan, A Kanik und U Atik (2004): Leptin and TNF-alpha levels in patients with chronic obstructive pulmonary disease and their relationship to nutritional parameters. Respiration 71, 45-50

Canalis E, G Mazziotti, A Giustina und JP Bilezikian (2007): Glucocorticoid-induced osteoporosis: pathophysiology and therapy. Osteoporos Int $\underline{18}, 1319-28$

Chua TP, P Ponikowski, K Webb-Peploe, D Harrington, SD Anker, M Piepoli und AJ Coats (1997): Clinical characteristics of chronic heart failure patients with an augmented peripheral chemoreflex. Eur Heart J 18, 480-6

Coats AJ, S Adamopoulos, A Radaelli, A McCance, TE Meyer, L Bernardi, PL Solda, P Davey, O Ormerod, C Forfar und et al. (1992): Controlled trial of physical training in chronic heart failure. Exercise performance, hemodynamics, ventilation, and autonomic function. Circulation 85, 2119-31

Cohn JN, TB Levine, MT Olivari, V Garberg, D Lura, GS Francis, AB Simon und T Rector (1984): Plasma norepinephrine as a guide to prognosis in patients with chronic congestive heart failure. N Engl J Med 311, 819-23 
Coirault C, A Hagege, D Chemla, MD Fratacci, C Guerot und Y Lecarpentier (2001): Angiotensinconverting enzyme inhibitor therapy improves respiratory muscle strength in patients with heart failure. Chest $\underline{119}, 1755-60$

Communal C, K Singh, DR Pimentel und WS Colucci (1998): Norepinephrine stimulates apoptosis in adult rat ventricular myocytes by activation of the beta-adrenergic pathway. Circulation $\underline{98}$, 1329-34

Costa F und I Biaggioni (1994): Role of adenosine in the sympathetic activation produced by isometric exercise in humans. J Clin Invest $\underline{93}, 1654-60$

Cote C, MD Zilberberg, SH Mody, LJ Dordelly und B Celli (2007): Haemoglobin level and its clinical impact in a cohort of patients with COPD. Eur Respir J 29, 923-9

Dalla Libera L, B Ravara, A Angelini, K Rossini, M Sandri, G Thiene, G Battista Ambrosio und G Vescovo (2001): Beneficial effects on skeletal muscle of the angiotensin II type 1 receptor blocker irbesartan in experimental heart failure. Circulation 103, 2195-200

Dauber IM und JV Weil (1983): Lung injury edema in dogs. Influence of sympathetic ablation. J Clin Invest $\underline{72}, 1977-86$

De Marco R, S Accordini, I Cerveri, A Corsico, J Sunyer, F Neukirch, N Kunzli, B Leynaert, C Janson, T Gislason, P Vermeire, C Svanes, JM Anto und P Burney (2004): An international survey of chronic obstructive pulmonary disease in young adults according to GOLD stages. Thorax $\underline{59}, 120-5$

De Matos LD, G Gardenghi, MU Rondon, HN Soufen, AP Tirone, AC Barretto, PC Brum, HR Middlekauff und CE Negrao (2004): Impact of 6 months of therapy with carvedilol on muscle sympathetic nerve activity in heart failure patients. J Card Fail $\underline{10}$, 496-502

Debigare R, CH Cote und F Maltais (2001): Peripheral muscle wasting in chronic obstructive pulmonary disease. Clinical relevance and mechanisms. Am J Respir Crit Care Med 164, $1712-7$

Dempsey JA, AW Sheel, CM St Croix und BJ Morgan (2002): Respiratory influences on sympathetic vasomotor outflow in humans. Respir Physiol Neurobiol 130, 3-20

Derchak PA, AW Sheel, BJ Morgan und JA Dempsey (2002): Effects of expiratory muscle work on muscle sympathetic nerve activity. J Appl Physiol 92, 1539-52

Donaldson GC, TA Seemungal, IS Patel, A Bhowmik, TM Wilkinson, JR Hurst, PK Maccallum und JA Wedzicha (2005): Airway and systemic inflammation and decline in lung function in patients with COPD. Chest $\underline{128}$, 1995-2004

Dransfield MT, SM Rowe, JE Johnson, WC Bailey und LB Gerald (2008): Use of beta blockers and the risk of death in hospitalised patients with acute exacerbations of COPD. Thorax 63, 301-5

Du YH und AF Chen (2007): A "love triangle" elicited by electrochemistry: complex interactions among cardiac sympathetic afferent, chemo-, and baroreflexes. J Appl Physiol 102, 9-10 
Duplain H, L Vollenweider, A Delabays, P Nicod, P Bartsch und U Scherrer (1999): Augmented sympathetic activation during short-term hypoxia and high-altitude exposure in subjects susceptible to high-altitude pulmonary edema. Circulation 99, 1713-8

Egred M, S Shaw, B Mohammad, P Waitt und E Rodrigues (2005): Under-use of beta-blockers in patients with ischaemic heart disease and concomitant chronic obstructive pulmonary disease. QJM 98, 493-7

European Respiratory Society (2006): Lifting the smoke screen - 10 reasons for a smoke free Europe. European Respiratory Society. Brussels, Belgium 2006

Engblom D, M Ek, S Saha, A Ericsson-Dahlstrand, PJ Jakobsson und A Blomqvist (2002): Prostaglandins as inflammatory messengers across the blood-brain barrier. J Mol Med (Berl) 80, 5-15

Engelhardt S, M Bohm, E Erdmann und MJ Lohse (1996): Analysis of beta-adrenergic receptor mRNA levels in human ventricular biopsy specimens by quantitative polymerase chain reactions: progressive reduction of beta 1-adrenergic receptor mRNA in heart failure. J Am Coll Cardiol 27, 146-54

Esler M (2002): Differentiation in the effects of the angiotensin II receptor blocker class on autonomic function. J Hypertens Suppl 20, S13-9

Fantuzzi G und R Faggioni (2000): Leptin in the regulation of immunity, inflammation, and hematopoiesis. J Leukoc Biol 68, 437-46

Feng Q, X Sun, X Lu, L Edvinsson und T Hedner (1999): Decreased responsiveness of vascular postjunctional alpha1-, alpha2-adrenoceptors and neuropeptide Y1 receptors in rats with heart failure. Acta Physiol Scand 166, 285-91

Ferguson DW, WJ Berg und JS Sanders (1990): Clinical and hemodynamic correlates of sympathetic nerve activity in normal humans and patients with heart failure: evidence from direct microneurographic recordings. J Am Coll Cardiol 16, 1125-34

Ferri CC und AV Ferguson (2005): Prostaglandin E2 mediates cellular effects of interleukin-1beta on parvocellular neurones in the paraventricular nucleus of the hypothalamus. J Neuroendocrinol 17, 498-508

Fischer BM, E Pavlisko und JA Voynow (2011): Pathogenic triad in COPD: oxidative stress, proteaseantiprotease imbalance, and inflammation. Int J Chron Obstruct Pulmon Dis $\underline{6}$, 413-21

Fisher LA (1993): Central actions of corticotropin-releasing factor on autonomic nervous activity and cardiovascular functioning. Ciba Found Symp 172, 243-53; discussion 253-7

Floras JS (2009): Sympathetic nervous system activation in human heart failure: clinical implications of an updated model. J Am Coll Cardiol 54, 375-85

Franchitto N, F Despas, M Labrunee, J Roncalli, S Boveda, M Galinier, JM Senard und A Pathak (2010): Tonic chemoreflex activation contributes to increased sympathetic nerve activity in heart failure-related anemia. Hypertension $\underline{55}, 1012-7$ 
Francis GS (1989): The relationship of the sympathetic nervous system and the renin-angiotensin system in congestive heart failure. Am Heart J $\underline{118}, 642-8$

Francis J, RM Weiss, AK Johnson und RB Felder (2003): Central mineralocorticoid receptor blockade decreases plasma TNF-alpha after coronary artery ligation in rats. Am J Physiol Regul Integr Comp Physiol 284, R328-35

Fu YC, CS Chi, SC Yin, B Hwang, YT Chiu und SL Hsu (2004): Norepinephrine induces apoptosis in neonatal rat cardiomyocytes through a reactive oxygen species-TNF alpha-caspase signaling pathway. Cardiovasc Res $\underline{62}$, 558-67

Garrido AM und KK Griendling (2009): NADPH oxidases and angiotensin II receptor signaling. Mol Cell Endocrinol 302, 148-58

Garrido PC, JM Diez, JR Gutierrez, AM Centeno, EG Vazquez, AG Miguel, MG Carballo und RJ Garcia (2006): Negative impact of chronic obstructive pulmonary disease on the health-related quality of life of patients. Results of the EPIDEPOC study. Health and Quality of Life Outcomes $\underline{4: 31}$

Gershon AS, L Warner, P Cascagnette, JC Victor und T To (2011): Lifetime risk of developing chronic obstructive pulmonary disease: a longitudinal population study Lancet $\underline{378}$, 991-6

Goldsmith SR (2004): Interactions between the sympathetic nervous system and the RAAS in heart failure. Curr Heart Fail Rep 1, 45-50

Gosker HR, NH Lencer, FM Franssen, GJ van der Vusse, EF Wouters und AM Schols (2003): Striking similarities in systemic factors contributing to decreased exercise capacity in patients with severe chronic heart failure or COPD. Chest $\underline{123}$, 1416-24

Goso Y, H Asanoi, H Ishise, T Kameyama, T Hirai, T Nozawa, S Takashima, K Umeno und H Inoue (2001): Respiratory modulation of muscle sympathetic nerve activity in patients with chronic heart failure. Circulation $\underline{104}, 418-23$

Graat-Verboom L, EF Wouters, FW Smeenk, BE van den Borne, R Lunde und MA Spruit (2009): Current status of research on osteoporosis in COPD: a systematic review. Eur Respir J $\underline{34}$, 209-18

Grassi G, G Seravalle, F Quarti-Trevano und R Dell'oro (2009): Sympathetic activation in congestive heart failure: evidence, consequences and therapeutic implications. Curr Vasc Pharmacol $\underline{7}$, $137-45$

Guggilam A, KP Patel, M Haque, PJ Ebenezer, DR Kapusta und J Francis (2008): Cytokine blockade attenuates sympathoexcitation in heart failure: cross-talk between nNOS, AT-1R and cytokines in the hypothalamic paraventricular nucleus. Eur J Heart Fail $\underline{10}$, 625-34

Halbert RJ, S Isonaka, D George und A Iqbal (2003): Interpreting COPD prevalence estimates: what is the true burden of disease?. Chest $123,1684-92$

Halbert RJ, JL Natoli, A Gano, E Badamgarav, AS Buist und DM Mannino (2006): Global burden of COPD: systematic review and meta-analysis. Eur Respir J 28, 523-32 
Hansell AL, JA Walk und JB Soriano (2003): What do chronic obstructive pulmonary disease patients die from? A multiple cause coding analysis. Eur Respir J 22, 809-14

Hansen J und M Sander (2003): Sympathetic neural overactivity in healthy humans after prolonged exposure to hypobaric hypoxia. J Physiol $\underline{546}, 921-9$

Hardy R und MS Cooper (2009): Bone loss in inflammatory disorders J Endocrinol 201, 309-20

Heindl S, C Dodt, M Krahwinkel, G Hasenfuss und S Andreas (2001a): Short term effect of continuous positive airway pressure on muscle sympathetic nerve activity in patients with chronic heart failure. Heart $\underline{85}$, 185-90

Heindl S, M Lehnert, CP Criee, G Hasenfuss und S Andreas (2001b): Marked sympathetic activation in patients with chronic respiratory failure. Am J Respir Crit Care Med 164, 597-601

Hill JM (2000): Discharge of group IV phrenic afferent fibers increases during diaphragmatic fatigue. Brain Res 856, 240-4

Hjalmarsen A, U Aasebo, G Aleksandersen und R Jorde (1996): Cardiovascular responses to tests for autonomic dysfunction in patients with chronic obstructive pulmonary disease with and without continuous long-term oxygen therapy. J Auton Nerv Syst $\underline{60}$, 169-74

Hnizdo E, PA Sullivan, KM Bang und G Wagner (2002): Association between chronic obstructive pulmonary disease and employment by industry and occupation in the US population: a study of data from the Third National Health and Nutrition Examination Survey. Am J Epidemiol $\underline{156}, 738-46$

Hofford JM, L Milakofsky, WH Vogel, RS Sacher, GJ Savage und S Pell (1990): The nutritional status in advanced emphysema associated with chronic bronchitis. A study of amino acid and catecholamine levels. Am Rev Respir Dis 141, 902-8

Hogg JC (2004): Pathophysiology of airflow limitation in chronic obstructive pulmonary disease. Lancet $\underline{364}, 709-21$

Huang BS und FH Leenen (2005): Blockade of brain mineralocorticoid receptors or $\mathrm{Na}+$ channels prevents sympathetic hyperactivity and improves cardiac function in rats post-MI. Am J Physiol Heart Circ Physiol 288, H2491-7

Hunt SA, WT Abraham, MH Chin, AM Feldman, GS Francis, TG Ganiats, M Jessup, MA Konstam, DM Mancini, K Michl (2005): ACC/AHA 2005 Guideline Update for the Diagnosis and Management of Chronic Heart Failure in the Adult: a report of the American College of Cardiology/American Heart Association Task Force on Practice Guidelines (Writing Committee to Update the 2001 Guidelines for the Evaluation and Management of Heart Failure): developed in collaboration with the American College of Chest Physicians and the International Society for Heart and Lung Transplantation: endorsed by the Heart Rhythm Society. Circulation 112, e154-235 
Janssen SP, G Gayan-Ramirez, A Van den Bergh, P Herijgers, K Maes, E Verbeken und M Decramer (2005): Interleukin-6 causes myocardial failure and skeletal muscle atrophy in rats. Circulation 111, 996-1005

Jennings GL (1998): Noradrenaline spillover and microneurography measurements in patients with primary hypertension. J Hypertens Suppl $\underline{16}$, S35-8

Jensen HH, NS Godtfredsen, P Lange und J Vestbo (2006): Potential misclassification of causes of death from COPD. Eur Respir J $\underline{28}$, 781-5

Jessup M und S Brozena (2003): Heart failure. N Engl J Med 348, 2007-18

John M, S Hoernig, W Doehner, DD Okonko, C Witt und SD Anker (2005): Anemia and inflammation in COPD. Chest $\underline{127}, 825-9$

Jones JG, BD Minty, P Lawler, G Hulands, JC Crawley und N Veall (1980): Increased alveolar epithelial permeability in cigarette smokers. Lancet 1980, 1, 66-8

Jung R, ME Dibner-Dunlap, MA Gilles und MD Thames (1995): Cardiorespiratory reflex control in rats with left ventricular dysfunction. Am J Physiol 268, H218-25

Kang YM, ZH Zhang, RF Johnson, Y Yu, T Beltz, AK Johnson, RM Weiss und RB Felder (2006): Novel effect of mineralocorticoid receptor antagonism to reduce proinflammatory cytokines and hypothalamic activation in rats with ischemia-induced heart failure. Circ Res $\underline{99}$, 758-66

Kaprielian RR, E Dupont, S Hafizi, PA Poole-Wilson, A Khaghani, MH Yacoub und NJ Severs (1997): Angiotensin II receptor type 1 mRNA is upregulated in atria of patients with end-stage heart failure. J Mol Cell Cardiol 29, 2299-304

Katz SD, K Hryniewicz, I Hriljac, K Balidemaj, C Dimayuga, A Hudaihed und A Yasskiy (2005): Vascular endothelial dysfunction and mortality risk in patients with chronic heart failure. Circulation 111, 310-4

Kawamura A, F Yuasa, H Yokoe, Y Masue, T Sugiura und T Iwasaka (2009): Augmented sympathoinhibitory effect of valsartan when added to angiotensin-converting enzyme inhibitor in patients with left ventricular dysfunction. J Cardiol $\underline{53}$, 171-8

Keatings VM, PD Collins, DM Scott und PJ Barnes (1996): Differences in interleukin-8 and tumor necrosis factor-alpha in induced sputum from patients with chronic obstructive pulmonary disease or asthma. Am J Respir Crit Care Med 153, 530-4

Kelly FJ und JC Fussell (2011): Air pollution and airway disease Clin Exp Allergy 41, 1059-71

Kenney MJ, ML Weiss und JR Haywood (2003): The paraventricular nucleus: an important component of the central neurocircuitry regulating sympathetic nerve outflow. Acta Physiol Scand $\underline{177}, 7-15$

Kleiber AC, H Zheng, HD Schultz, JD Peuler und KP Patel (2008): Exercise training normalizes enhanced glutamate-mediated sympathetic activation from the PVN in heart failure. Am J Physiol Regul Integr Comp Physiol 294, R1863-72 
Koehler F, W Doehner, S Hoernig, C Witt, SD Anker und M John (2007): Anorexia in chronic obstructive pulmonary disease--association to cachexia and hormonal derangement. Int $\mathrm{J}$ Cardiol 119, 83-9

Kon H, M Nakamura, N Arakawa und K Hiramori (2004): Muscle metaboreflex is blunted with reduced vascular resistance response of nonexercised limb in patients with chronic heart failure. J Card Fail 10, 503-10

Krum H und JR Teerlink (2011): Medical therapy for chronic heart failure. Lancet $\underline{378}$, 713-21

Lahiri MK, PJ Kannankeril und JJ Goldberger (2008): Assessment of autonomic function in cardiovascular disease: physiological basis and prognostic implications. J Am Coll Cardiol $\underline{51}$, 1725-33

Lal A, JP Veinot und FH Leenen (2004): Critical role of CNS effects of aldosterone in cardiac remodeling post-myocardial infarction in rats. Cardiovasc Res 64, 437-47

Lal A, JP Veinot, D Ganten und FH Leenen (2005): Prevention of cardiac remodeling after myocardial infarction in transgenic rats deficient in brain angiotensinogen. J Mol Cell Cardiol 39, 521-9

Lambert C, Y Massillon und S Meloche (1995): Upregulation of cardiac angiotensin II AT1 receptors in congenital cardiomyopathic hamsters. Circ Res $\underline{77}$, 1001-7

Leenen FH (2007): Brain mechanisms contributing to sympathetic hyperactivity and heart failure. Circ Res 101, 221-3

Leenen FH, BS Huang, H Yu und B Yuan (1995): Brain 'ouabain' mediates sympathetic hyperactivity in congestive heart failure. Circ Res 77, 993-1000

Lehouck A, S Boonen, M Decramer und W Janssens (2011): COPD, bone metabolism, and osteoporosis. Chest $\underline{139}, 648-57$

Levine S, T Nguyen, LR Kaiser, NA Rubinstein, G Maislin, C Gregory, LC Rome, GA Dudley, GC Sieck und JB Shrager (2003): Human diaphragm remodeling associated with chronic obstructive pulmonary disease: clinical implications. Am J Respir Crit Care Med 168, 706-13

Li YF, W Wang, WG Mayhan und KP Patel (2006): Angiotensin-mediated increase in renal sympathetic nerve discharge within the PVN: role of nitric oxide. Am J Physiol Regul Integr Comp Physiol 290, R1035-43

Li YL, Y Ding, C Agnew und HD Schultz (2008): Exercise training improves peripheral chemoreflex function in heart failure rabbits. J Appl Physiol 105, 782-90

Lin R, H Peng, LP Nguyen, NB Dudekula, F Shardonofsky, BJ Knoll, S Parra und RA Bond (2008): Changes in beta 2-adrenoceptor and other signaling proteins produced by chronic administration of 'beta-blockers' in a murine asthma model. Pulm Pharmacol Ther 21, 115-24

Ling Y, W Saleem und CD Shee (2008): Concomitant use of beta-blockers and beta2-agonists. Eur Respir J 31, 905-6 
Liu JL, S Irvine, IA Reid, KP Patel und IH Zucker (2000): Chronic exercise reduces sympathetic nerve activity in rabbits with pacing-induced heart failure: A role for angiotensin II. Circulation 102, 1854-62

Llorens-Cortes C, B Greenberg, H Huang und P Corvol (1994): Tissular expression and regulation of type 1 angiotensin II receptor subtypes by quantitative reverse transcriptase-polymerase chain reaction analysis. Hypertension 24, 538-48

Lokke A, P Lange, H Scharling, P Fabricius und J Vestbo (2006): Developing COPD: a 25 year follow up study of the general population. Thorax $\underline{61}$, 935-9

Lopez AD, K Shibuya, C Rao, CD Mathers, AL Hansell, LS Held, V Schmid und S Buist (2006): Chronic obstructive pulmonary disease: current burden and future projections. Eur Respir J 27, 397-412

Machado BH, H Mauad, DA Chianca Junior, AS Haibara und E Colombari (1997): Autonomic processing of the cardiovascular reflexes in the nucleus tractus solitarii. Braz J Med Biol Res 30, 533-43

Mador MJ (2002): Muscle mass, not body weight, predicts outcome in patients with chronic obstructive pulmonary disease. Am J Respir Crit Care Med 166, 787-9

Maestrelli P, C Paska, M Saetta, G Turato, Y Nowicki, S Monti, B Formichi, M Miniati und LM Fabbri (2003): Decreased haem oxygenase-1 and increased inducible nitric oxide synthase in the lung of severe COPD patients. Eur Respir J 21, 971-6

Malli F, AI Papaioannou, KI Gourgoulianis und Z Daniil (2010): The role of leptin in the respiratory system: an overview. Respir Res $\underline{11}, 152$

Malliani A und N Montano (2002): Emerging excitatory role of cardiovascular sympathetic afferents in pathophysiological conditions. Hypertension $\underline{39}$, 63-8

Mancini GB (2005): The 'double dip' hypothesis: simultaneous prevention of cardiovascular and pulmonary morbidity and mortality using angiotensin II type 1 receptor blockers. Can J Cardiol 21, 519-23

Mancini GB, M Etminan, B Zhang, LE Levesque, JM FitzGerald und JM Brophy (2006): Reduction of morbidity and mortality by statins, angiotensin-converting enzyme inhibitors, and angiotensin receptor blockers in patients with chronic obstructive pulmonary disease. J Am Coll Cardiol 47, 2554-60

Mann DL, RL Kent, B Parsons und Gt Cooper (1992): Adrenergic effects on the biology of the adult mammalian cardiocyte. Circulation $\underline{85}$, 790-804

Mannino DM, D Thorn, A Swensen und F Holguin (2008): Prevalence and outcomes of diabetes, hypertension and cardiovascular disease in COPD. Eur Respir J 32, 962-9

Mark AL, RG Victor, C Nerhed und BG Wallin (1985): Microneurographic studies of the mechanisms of sympathetic nerve responses to static exercise in humans. Circ Res $\underline{57}, 461-9$ 
Marshall JM (1994): Peripheral chemoreceptors and cardiovascular regulation. Physiol Rev 74, 54394

Mead J, JM Turner, PT Macklem und JB Little (1967): Significance of the relationship between lung recoil and maximum expiratory flow. J Appl Physiol 22, 95-108

Mitchell JH, MP Kaufman und GA Iwamoto (1983): The exercise pressor reflex: its cardiovascular effects, afferent mechanisms, and central pathways. Annu Rev Physiol 45, 229-42

Molfino NA (2004): Genetics of COPD Chest $\underline{125}$, 1929-40

Molteni A, WF Ward, CH Ts'ao, J Taylor, W Small, Jr., L Brizio-Molteni und PA Veno (2003): Cytostatic properties of some angiotensin I converting enzyme inhibitors and of angiotensin II type I receptor antagonists. Curr Pharm Des $\underline{9}$, 751-61

Munhoz RT, CE Negrao, AC Barretto, ME Ochiai, JN Cardoso, PC Morgado, CH Del Carlo und JA Ramires (2009): Microneurography and venous occlusion plethysmography in heart failure: correlation with prognosis. Arq Bras Cardiol 92, 46-53

Murray CJ und AD Lopez (1997): Alternative projections of mortality and disability by cause 19902020: Global Burden of Disease Study. Lancet 349, 1498-504

Narkiewicz K, CA Pesek, PJ van de Borne, M Kato und VK Somers (1999): Enhanced sympathetic and ventilatory responses to central chemoreflex activation in heart failure. Circulation $\underline{100}$, $262-7$

National Heart L, and Blood Institute (1985): The definition of emphysema. Report of a National Heart, Lung, and Blood Institute, Division of Lung Diseases workshop. Am Rev Respir Dis $\underline{132}, 182-5$

Navas EV und DO Taylor (2010): Q: Can patients with COPD or asthma take a beta-blocker?. Cleve Clin J Med 77, 498-9

Negrao CE, MU Rondon, T Tinucci, MJ Alves, F Roveda, AM Braga, SF Reis, L Nastari, AC Barretto, EM Krieger und HR Middlekauff (2001): Abnormal neurovascular control during exercise is linked to heart failure severity. Am J Physiol Heart Circ Physiol 280, H1286-92

Neri M, D Cerretani, AI Fiaschi, PF Laghi, PE Lazzerini, AB Maffione, L Micheli, G Bruni, C Nencini, G Giorgi, S D'Errico, C Fiore, C Pomara, I Riezzo, E Turillazzi und V Fineschi (2007): Correlation between cardiac oxidative stress and myocardial pathology due to acute and chronic norepinephrine administration in rats. J Cell Mol Med 11, 156-70

Nio Y, H Matsubara, S Murasawa, M Kanasaki und M Inada (1995): Regulation of gene transcription of angiotensin II receptor subtypes in myocardial infarction. J Clin Invest $\underline{95}$, 46-54

Nishikawa M, JC Mak, H Shirasaki, SE Harding und PJ Barnes (1994): Long-term exposure to norepinephrine results in down-regulation and reduced mRNA expression of pulmonary betaadrenergic receptors in guinea pigs. Am J Respir Cell Mol Biol 10, 91-9 
Notarius CF, DJ Atchison, GA Rongen und JS Floras (2001): Effect of adenosine receptor blockade with caffeine on sympathetic response to handgrip exercise in heart failure. Am J Physiol Heart Circ Physiol 281, H1312-8

Notarius CF, J Spaak, BL Morris und JS Floras (2007): Comparison of muscle sympathetic activity in ischemic and nonischemic heart failure. J Card Fail $\underline{13}$, 470-5

Olivetti G, R Abbi, F Quaini, J Kajstura, W Cheng, JA Nitahara, E Quaini, C Di Loreto, CA Beltrami, S Krajewski, JC Reed und P Anversa (1997): Apoptosis in the failing human heart. N Engl J Med 336, 1131-41

Orozco-Levi M, J Lloreta, J Minguella, S Serrano, JM Broquetas und J Gea (2001): Injury of the human diaphragm associated with exertion and chronic obstructive pulmonary disease. Am J Respir Crit Care Med 164, 1734-9

Ottenheijm CA, LM Heunks und PN Dekhuijzen (2007): Diaphragm muscle fiber dysfunction in chronic obstructive pulmonary disease: toward a pathophysiological concept. Am J Respir Crit Care Med $\underline{175}, 1233-40$

Ottenheijm CA, LM Heunks und RP Dekhuijzen (2008): Diaphragm adaptations in patients with COPD. Respir Res $\underline{9}, 12$

Packer M (1992): The neurohormonal hypothesis: a theory to explain the mechanism of disease progression in heart failure. J Am Coll Cardiol 20, 248-54

Park HY und D Sin (2011): Smoking kills, quitting heals: the importance of smoking cessation in COPD. Clin Respir J $\underline{5}, 185-6$

Patakas D, G Louridas und E Kakavelas (1982): Reduced baroreceptor sensitivity in patients with chronic obstructive pulmonary disease. Thorax $\underline{37}, 292-5$

Patel KP und H Zheng (2012): Central neural control of sympathetic nerve activity in heart failure following exercise training. Am J Physiol Heart Circ Physiol 302, H527-37

Piepoli M, AL Clark, M Volterrani, S Adamopoulos, P Sleight und AJ Coats (1996): Contribution of muscle afferents to the hemodynamic, autonomic, and ventilatory responses to exercise in patients with chronic heart failure: effects of physical training. Circulation $\underline{93}$, 940-52

Ponikowski P, SD Anker, TP Chua, R Szelemej, M Piepoli, S Adamopoulos, K Webb-Peploe, D Harrington, W Banasiak, K Wrabec und AJ Coats (1997a): Depressed heart rate variability as an independent predictor of death in chronic congestive heart failure secondary to ischemic or idiopathic dilated cardiomyopathy. Am J Cardiol 79, 1645-50

Ponikowski P, TP Chua, M Piepoli, D Ondusova, K Webb-Peploe, D Harrington, SD Anker, M Volterrani, R Colombo, G Mazzuero, A Giordano und AJ Coats (1997b): Augmented peripheral chemosensitivity as a potential input to baroreflex impairment and autonomic imbalance in chronic heart failure. Circulation 96, 2586-94

Rabe KF, S Hurd, A Anzueto, PJ Barnes, SA Buist, P Calverley, Y Fukuchi, C Jenkins, R RodriguezRoisin, C van Weel und J Zielinski (2007): Global strategy for the diagnosis, management, 
and prevention of chronic obstructive pulmonary disease: GOLD executive summary. Am J Respir Crit Care Med 176, 532-55

Raherison C und PO Girodet (2009): Epidemiology of COPD. Eur Respir Rev 18, 213-21

Rahman I, SK Biswas und A Kode (2006): Oxidant and antioxidant balance in the airways and airway diseases. Eur J Pharmacol 533, 222-39

Raupach T, F Bahr, P Herrmann, L Luethje, K Heusser, G Hasenfuss, L Bernardi und S Andreas (2008): Slow breathing reduces sympathoexcitation in COPD. Eur Respir J $\underline{32}$, 387-92

Raupach T, F Bahr, P Herrmann, L Luthje, G Hasenfuss und S Andreas (2010): Inspiratory resistive loading does not increase sympathetic tone in COPD. Respir Med 104, 107-13

Raupach T, L Luthje, H Kogler, C Duve, F Schweda, G Hasenfuss und S Andreas (2011): Local and systemic effects of angiotensin receptor blockade in an emphysema mouse model. Pulm Pharmacol Ther 24, 215-20

Reid IA (1992): Interactions between ANG II, sympathetic nervous system, and baroreceptor reflexes in regulation of blood pressure. Am J Physiol 262, E763-78

Rennard SI und J Vestbo (2006): COPD: the dangerous underestimate of 15\% Lancet 367, 1216-9

Rivest S, S Lacroix, L Vallieres, S Nadeau, J Zhang und N Laflamme (2000): How the blood talks to the brain parenchyma and the paraventricular nucleus of the hypothalamus during systemic inflammatory and infectious stimuli. Proc Soc Exp Biol Med 223, 22-38

Rodman JR, KS Henderson, CA Smith und JA Dempsey (2003): Cardiovascular effects of the respiratory muscle metaboreflexes in dogs: rest and exercise. J Appl Physiol 95, 1159-69

Rodrigues TC, J Ehrlich, CM Hunter, GL Kinney, M Rewers und JK Snell-Bergeon (2010): Reduced heart rate variability predicts progression of coronary artery calcification in adults with type 1 diabetes and controls without diabetes. Diabetes Technol Ther $\underline{12}$, 963-9

Roseguini BT, CN Alves, GR Chiappa, R Stein, MM Knorst und JP Ribeiro (2008): Attenuation of muscle metaboreflex in chronic obstructive pulmonary disease. Med Sci Sports Exerc 40, 9-14

Roveda F, HR Middlekauff, MU Rondon, SF Reis, M Souza, L Nastari, AC Barretto, EM Krieger und CE Negrao (2003): The effects of exercise training on sympathetic neural activation in advanced heart failure: a randomized controlled trial. J Am Coll Cardiol 42, 854-60

Rutten FH, NP Zuithoff, E Hak, DE Grobbee und AW Hoes (2010): Beta-blockers may reduce mortality and risk of exacerbations in patients with chronic obstructive pulmonary disease. Arch Intern Med $\underline{170}$, 880-7

Sakamaki F, T Satoh, N Nagaya, S Kyotani, N Nakanishi und Y Ishida (1999): Abnormality of left ventricular sympathetic nervous function assessed by (123)I-metaiodobenzylguanidine imaging in patients with COPD. Chest $\underline{116}, 1575-81$

Sala-Mercado JA, RL Hammond, JK Kim, PJ McDonald, LW Stephenson und DS O'Leary (2007): Heart failure attenuates muscle metaboreflex control of ventricular contractility during dynamic exercise. Am J Physiol Heart Circ Physiol 292, H2159-66 
Salpeter S, T Ormiston und E Salpeter (2002a): Cardioselective beta-blockers for reversible airway disease. Cochrane Database Syst Rev, CD002992

Salpeter S, T Ormiston, E Salpeter, P Poole und C Cates (2002b): Cardioselective beta-blockers for chronic obstructive pulmonary disease. Cochrane Database Syst Rev, CD003566

Scalvini S, R Porta, E Zanelli, M Volterrani, M Vitacca, M Pagani, A Giordano und N Ambrosino (1999): Effects of oxygen on autonomic nervous system dysfunction in patients with chronic obstructive pulmonary disease. Eur Respir J $\underline{13}, 119-24$

Schols AM, EC Creutzberg, WA Buurman, LA Campfield, WH Saris und EF Wouters (1999): Plasma leptin is related to proinflammatory status and dietary intake in patients with chronic obstructive pulmonary disease. Am J Respir Crit Care Med 160, 1220-6

Schonhofer B, H Bohrer und D Kohler (1998a): Blood transfusion facilitating difficult weaning from the ventilator. Anaesthesia 53, 181-4

Schonhofer B, M Wenzel, M Geibel und D Kohler (1998b): Blood transfusion and lung function in chronically anemic patients with severe chronic obstructive pulmonary disease. Crit Care Med 26, $1824-8$

Scott AC, R Wensel, CH Davos, M Kemp, A Kaczmarek, J Hooper, AJ Coats und MF Piepoli (2002): Chemical mediators of the muscle ergoreflex in chronic heart failure: a putative role for prostaglandins in reflex ventilatory control. Circulation $\underline{106}$, 214-20

Scott AC, R Wensel, CH Davos, P Georgiadou, M Kemp, J Hooper, AJ Coats und MF Piepoli (2003): Skeletal muscle reflex in heart failure patients: role of hydrogen. Circulation 107, 300-6

Seals DR (1989): Sympathetic neural discharge and vascular resistance during exercise in humans. J Appl Physiol 66, 2472-8

Seals DR und RG Victor (1991): Regulation of muscle sympathetic nerve activity during exercise in humans. Exerc Sport Sci Rev 19, 313-49

Seals DR und FA Dinenno (2004): Collateral damage: cardiovascular consequences of chronic sympathetic activation with human aging Am J Physiol Heart Circ Physiol 287, H1895-905

Seals DR, NO Suwarno und JA Dempsey (1990): Influence of lung volume on sympathetic nerve discharge in normal humans. Circ Res $\underline{67}$, 130-41

Seemungal TA, Hurst JR und Wedzicha, JA (2009): Exacerbation rate, health status and mortality in COPD- a review of potential interventions. Int J Chron Obstruct Pulm Dis $\underline{4}$, 203-23

Sergi G, A Coin, S Marin, A Vianello, A Manzan, S Peruzza, EM Inelmen, L Busetto, S Mulone und G Enzi (2006): Body composition and resting energy expenditure in elderly male patients with chronic obstructive pulmonary disease. Respir Med 100, 1918-24

Sethi S und TF Murphy (2001): Bacterial infection in chronic obstructive pulmonary disease in 2000: a state-of-the-art review. Clin Microbiol Rev 14, 336-63 
Shi Z, XB Gan, ZD Fan, F Zhang, YB Zhou, XY Gao, W De und GQ Zhu (2011): Inflammatory cytokines in paraventricular nucleus modulate sympathetic activity and cardiac sympathetic afferent reflex in rats. Acta Physiol (Oxf) 203, 289-97

Shields RW, Jr. (1993): Functional anatomy of the autonomic nervous system. J Clin Neurophysiol $\underline{10}$, $2-13$

Short PM, SI Lipworth, DH Elder, S Schembri und BJ Lipworth (2011): Effect of beta blockers in treatment of chronic obstructive pulmonary disease: a retrospective cohort study. BMJ $\underline{342}$, d2549

Sin DD, L Wu und SF Man (2005): The relationship between reduced lung function and cardiovascular mortality: a population-based study and a systematic review of the literature. Chest 127, 1952-9

Sin DD, NR Anthonisen, JB Soriano und AG Agusti (2006): Mortality in COPD: Role of comorbidities. Eur Respir J 28, 1245-57

Snitker S, RE Pratley, M Nicolson, PA Tataranni und E Ravussin (1997): Relationship between muscle sympathetic nerve activity and plasma leptin concentration. Obes Res $\underline{5}$, 338-40

Snitker S, PA Tataranni und E Ravussin (1998): Respiratory quotient is inversely associated with muscle sympathetic nerve activity. J Clin Endocrinol Metab 83, 3977-9

Soler-Cataluna JJ, Martinez-Garcia MA, Roman Sanchez P, Salcedo E, Navarro M und Ochando R (2005): Severe acute exacerbations and mortality in patients with chronic obstructive pulmonary disease. Thorax 60 , 925-31

Somers VK, AL Mark, DC Zavala und FM Abboud (1989): Influence of ventilation and hypocapnia on sympathetic nerve responses to hypoxia in normal humans. J Appl Physiol 67, 2095-100

Somers VK, AL Mark und FM Abboud (1991): Interaction of baroreceptor and chemoreceptor reflex control of sympathetic nerve activity in normal humans. J Clin Invest $\underline{87}$, 1953-7

Spaak J, ZJ Egri, T Kubo, E Yu, S Ando, Y Kaneko, K Usui, TD Bradley und JS Floras (2005): Muscle sympathetic nerve activity during wakefulness in heart failure patients with and without sleep apnea. Hypertension $\underline{46}$, 1327-32

Spruit MA, R Gosselink, T Troosters, A Kasran, G Gayan-Ramirez, P Bogaerts, R Bouillon und M Decramer (2003): Muscle force during an acute exacerbation in hospitalised patients with COPD and its relationship with CXCL8 and IGF-I. Thorax $\underline{58}$, 752-6

Stewart AG, JC Waterhouse, CG Billings, P Baylis und P Howard (1994): Effects of angiotensin converting enzyme inhibition on sodium excretion in patients with hypoxaemic chronic obstructive pulmonary disease. Thorax $\underline{49}$, 995-8

Stoller JK und LS Aboussouan (2005): Alpha1-antitrypsin deficiency. Lancet 365, 2225-36

Supinski GS und LA Callahan (2007): Free radical-mediated skeletal muscle dysfunction in inflammatory conditions. J Appl Physiol 102, 2056-63 
Suzuki J, H Matsubara, M Urakami und M Inada (1993): Rat angiotensin II (type 1A) receptor mRNA regulation and subtype expression in myocardial growth and hypertrophy. Circ Res $\underline{73}$, 439-47

Takabatake N, H Nakamura, O Minamihaba, M Inage, S Inoue, S Kagaya, M Yamaki und H Tomoike (2001): A novel pathophysiologic phenomenon in cachexic patients with chronic obstructive pulmonary disease: the relationship between the circadian rhythm of circulating leptin and the very low-frequency component of heart rate variability. Am J Respir Crit Care Med $\underline{163}$, $1314-9$

Tanni SE, NR Pelegrino, AY Angeleli, C Correa und I Godoy (2010): Smoking status and tumor necrosis factor-alpha mediated systemic inflammation in COPD patients. J Inflamm (Lond) 7 , 29

Testelmans D, T Crul, K Maes, A Agten, M Crombach, M Decramer und G Gayan-Ramirez (2010): Atrophy and hypertrophy signalling in the diaphragm of patients with COPD. Eur Respir J 35 , 549-56

Tjen ALS, A Bonham und J Longhurst (1997): Interactions between sympathetic and vagal cardiac afferents in nucleus tractus solitarii. Am J Physiol 272, H2843-51

Todd GL, G Baroldi, GM Pieper, FC Clayton und RS Eliot (1985): Experimental catecholamineinduced myocardial necrosis. II. Temporal development of isoproterenol-induced contraction band lesions correlated with ECG, hemodynamic and biochemical changes. J Mol Cell Cardiol 17, 647-56

Tracey KJ, H Wei, KR Manogue, Y Fong, DG Hesse, HT Nguyen, GC Kuo, B Beutler, RS Cotran, A Cerami und et al. (1988): Cachectin/tumor necrosis factor induces cachexia, anemia, and inflammation. J Exp Med 167, 1211-27

Triposkiadis F, G Karayannis, G Giamouzis, J Skoularigis, G Louridas und J Butler (2009): The sympathetic nervous system in heart failure physiology, pathophysiology, and clinical implications. J Am Coll Cardiol 노, 1747-62

Tsuji H, MG Larson, FJ Venditti, Jr., ES Manders, JC Evans, CL Feldman und D Levy (1996): Impact of reduced heart rate variability on risk for cardiac events. The Framingham Heart Study. Circulation $\underline{94}$, 2850-5

Tuder RM, BE Flook und NF Voelkel (1995): Increased gene expression for VEGF and the VEGF receptors KDR/Flk and Flt in lungs exposed to acute or to chronic hypoxia. Modulation of gene expression by nitric oxide. J Clin Invest $\underline{95}$, 1798-807

Turnbull AV und CL Rivier (1999): Regulation of the hypothalamic-pituitary-adrenal axis by cytokines: actions and mechanisms of action. Physiol Rev $\underline{79}, 1-71$

Vallbo AB, KE Hagbarth und BG Wallin (2004): Microneurography: how the technique developed and its role in the investigation of the sympathetic nervous system. J Appl Physiol 96, 1262-9

Viegi G, F Pistelli, DL Sherrill, S Maio, S Baldacci und L Carrozzi (2007): Definition, epidemiology and natural history of COPD. Eur Respir J 30, 993-1013 
Volterrani M, S Scalvini, G Mazzuero, P Lanfranchi, R Colombo, AL Clark und G Levi (1994): Decreased heart rate variability in patients with chronic obstructive pulmonary disease. Chest 106, $1432-7$

Wagner PD (2008): Possible mechanisms underlying the development of cachexia in COPD. Eur Respir J $\underline{31}, 492-501$

Wang H, BS Huang, D Ganten und FH Leenen (2004): Prevention of sympathetic and cardiac dysfunction after myocardial infarction in transgenic rats deficient in brain angiotensinogen. Circ Res $\underline{94}, 843$

Wang R, A Zagariya, O Ibarra-Sunga, C Gidea, E Ang, S Deshmukh, G Chaudhary, J Baraboutis, G Filippatos und BD Uhal (1999): Angiotensin II induces apoptosis in human and rat alveolar epithelial cells. Am J Physiol 276, L885-9

Wang W, JS Chen und IH Zucker (1990): Carotid sinus baroreceptor sensitivity in experimental heart failure. Circulation $\underline{81}$, 1959-66

Wang W, JS Chen und IH Zucker (1991): Carotid sinus baroreceptor reflex in dogs with experimental heart failure. Circ Res $\underline{68}$, 1294-301

Wang W, GQ Zhu, L Gao, W Tan und ZM Qian (2004): Baroreceptor reflex in heart failure. Sheng Li Xue Bao 56, 269-81

Watson AM, SG Hood und CN May (2006): Mechanisms of sympathetic activation in heart failure. Clin Exp Pharmacol Physiol 33, 1269-74

Weiss ST und S Shore (2004): Obesity and asthma: directions for research. Am J Respir Crit Care Med $\underline{169}, 963-8$

White CW (1981): Abnormalities in baroreflex control of heart rate in canine heart failure. Am J Physiol 240, H793-9

Witt JD, JA Guenette, JL Rupert, DC McKenzie und AW Sheel (2007): Inspiratory muscle training attenuates the human respiratory muscle metaboreflex. J Physiol $\underline{584}$, 1019-28

Wosten-van Asperen RM, R Lutter, JJ Haitsma, MP Merkus, JB van Woensel, CM van der Loos, S Florquin, B Lachmann und AP Bos (2008): ACE mediates ventilator-induced lung injury in rats via angiotensin II but not bradykinin. Eur Respir J $\underline{31}$, 363-71

Wright JL, T Petty und WM Thurlbeck (1992): Analysis of the structure of the muscular pulmonary arteries in patients with pulmonary hypertension and COPD: National Institutes of Health nocturnal oxygen therapy trial. Lung $\underline{170}, 109-24$

Wright JW und JW Harding (1992): Regulatory role of brain angiotensins in the control of physiological and behavioral responses. Brain Res Brain Res Rev $\underline{17}$, 227-62

Yin P, CQ Jiang, KK Cheng, TH Lam, KH Lam, MR Miller, WS Zhang, GN Thomas und P Adab (2007): Passive smoking exposure and risk of COPD among adults in China: the Guangzhou Biobank Cohort Study. Lancet $\underline{370}, 751-7$ 
Zarkesh-Esfahani H, AG Pockley, Z Wu, PG Hellewell, AP Weetman und RJ Ross (2004): Leptin indirectly activates human neutrophils via induction of TNF-alpha. J Immunol $\underline{172}$, 1809-14

Zhang ZH, J Francis, RM Weiss und RB Felder (2002): The renin-angiotensin-aldosterone system excites hypothalamic paraventricular nucleus neurons in heart failure. Am J Physiol Heart Circ Physiol 283, H423-33

Zhong S, SY Zhou, GL Gebber und SM Barman (1997): Coupled oscillators account for the slow rhythms in sympathetic nerve discharge and phrenic nerve activity. Am J Physiol 272, R131424

Zhu GQ, L Gao, Y Li, KP Patel, IH Zucker und W Wang (2004): AT1 receptor mRNA antisense normalizes enhanced cardiac sympathetic afferent reflex in rats with chronic heart failure. Am J Physiol Heart Circ Physiol 287, H1828-35 


\section{Danksagung}

Mein Dank gilt zunächst dem Direktor der Abteilung Kardiologie und Pneumologie der Medizinischen Fakultät der Georg-August-Universität Göttingen, Herrn Prof. Dr. med. Gerd Hasenfuß, für die Möglichkeit, die vorliegende Dissertation in seiner Abteilung durchzuführen.

Mein ganz besonderer Dank gilt Herrn Prof. Dr. med. Stefan Andreas für die Überlassung des interessanten und spannenden Themas, die exzellente Betreuung und für die vielen wertvollen Anregungen und Diskussionen.

Herrn Priv.-Doz. Dr. med. Tobias Raupach und Frau Priv.-Doz. Dr. med. Silke Heindl danke ich für die freundliche Unterstützung und für die mir zur Verfügung gestellten Studiendaten, ohne die meine Arbeit nicht möglich gewesen wäre. 


\section{Lebenslauf}

Am 27.09.1985 wurde ich, Stephan Frederik Klarner, als Sohn der Eheleute Dr. med. dent. Wilhelm Hans Klarner und Dr. med. Asta Monika Maria Klarner, geb. Sommer, in Warburg geboren.

Von 1992 bis 1996 besuchte ich die Mittelpunktschule Wilhelmsthal in Calden und wechselte anschließend auf das Engelsburg-Gymnasium in Kassel, wo ich am 16.06.2005 das Zeugnis der allgemeinen Hochschulreife erwarb.

Im Anschluss leistete ich vom 01.09.2005 bis 31.05.2005 meinen Zivildienst in der Paracelsus-ElenaKlinik in Kassel ab.

Im Wintersemester 2006/2007 begann ich das Studium der Humanmedizin an der Georg-AugustUniversität Göttingen. Am 09.09.2008 bestand ich den Ersten Abschnitt der Ärztlichen Prüfung. Mein Praktisches Jahr mit dem Wahlfach Mund-Kiefer-Gesichtschirurgie leiste ich derzeit an der Universitätsmedizin Göttingen, der Lungenfachklinik Immenhausen und am Evangelischen Krankenhaus Göttingen-Weende ab. 